\title{
Article
}

\section{A combined microRNA-based targeted therapeutic approach to eradicate glioblastoma stem-Iike cells}

Esposito, Carla L., Nuzzo, Silvia, Kumar, Swati A., Rienzo, Anna, Lawrence, Clare Louise, Pallini, Roberto, Shaw, Lisa, Alder, Jane Elizabeth, Ricci-Vitiani, Lucia, Catuogno, Silvia and De Franciscis, Vittorio

Available at http://clok.uclan.ac.uk/16805/

Esposito, Carla L., Nuzzo, Silvia, Kumar, Swati A., Rienzo, Anna, Lawrence, Clare Louise ORCID: 0000-0003-0170-0079, Pallini, Roberto, Shaw, Lisa ORCID: 0000-0002-6226-6467, Alder, Jane Elizabeth ORCID: 0000-0003-44630349, Ricci-Vitiani, Lucia et al (2016) A combined microRNA-based targeted therapeutic approach to eradicate glioblastoma stem-like cells. Journal of Controlled Release, 238 . pp. 43-57. ISSN 0168-3659

It is advisable to refer to the publisher's version if you intend to cite from the work. http://dx.doi.org/10.1016/j.jconrel.2016.07.032

For more information about UCLan's research in this area go to http://www.uclan.ac.uk/researchgroups/ and search for < name of research Group>.

For information about Research generally at UCLan please go to http://www.uclan.ac.uk/research/

All outputs in CLoK are protected by Intellectual Property Rights law, including Copyright law. Copyright, IPR and Moral Rights for the works on this site are retained by the individual authors and/or other copyright owners. Terms and conditions for use of this material are defined in the policies page. 


\section{Accepted Manuscript}

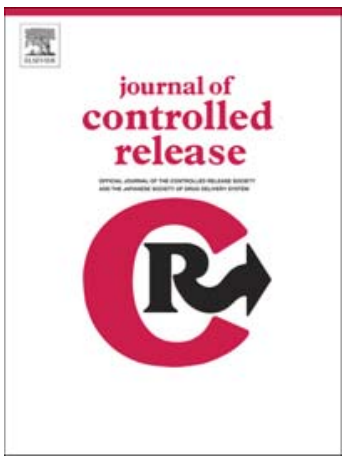

A combined microRNA-based targeted therapeutic approach to eradicate glioblastoma stem-like cells

Carla L. Esposito, Silvia Nuzzo, Swati A. Kumar, Anna Rienzo, Clare L. Lawrence, Roberto Pallini, Lisa Shaw, Jane E. Alder, Lucia Ricci-Vitiani, Silvia Catuogno, Vittorio de Franciscis

PII:

S0168-3659(16)30466-7

DOI:

doi: $10.1016 /$ j.jconrel.2016.07.032

Reference:

COREL 8389

To appear in: $\quad$ Journal of Controlled Release

Received date: 3 June 2016

Revised date: $\quad 18$ July 2016

Accepted date: 19 July 2016

Please cite this article as: Carla L. Esposito, Silvia Nuzzo, Swati A. Kumar, Anna Rienzo, Clare L. Lawrence, Roberto Pallini, Lisa Shaw, Jane E. Alder, Lucia Ricci-Vitiani, Silvia Catuogno, Vittorio de Franciscis, A combined microRNA-based targeted therapeutic approach to eradicate glioblastoma stem-like cells, Journal of Controlled Release (2016), doi: $10.1016 /$ j.jconrel.2016.07.032

This is a PDF file of an unedited manuscript that has been accepted for publication. As a service to our customers we are providing this early version of the manuscript. The manuscript will undergo copyediting, typesetting, and review of the resulting proof before it is published in its final form. Please note that during the production process errors may be discovered which could affect the content, and all legal disclaimers that apply to the journal pertain. 
A combined microRNA-based targeted therapeutic approach to eradicate glioblastoma stem-like cells

Carla L. Esposito', Silvia Nuzzo ${ }^{1}$, Swati A. Kumar², Anna Rienzo', Clare L. Lawrence ${ }^{2}$, Roberto Pallini $^{3}$, Lisa Shaw ${ }^{2}$, Jane E. Alder ${ }^{2}$, Lucia Ricci-Vitiani ${ }^{4}$, Silvia Catuogno ${ }^{1}$ and Vittorio de Franciscis $^{1^{*}}$

1 Istituto di Endocrinologia ed Oncologia Sperimentale "G. Salvatore", CNR, Naples, Italy

2 School of Pharmacy \& Biomedical Sciences, University of Central Lancashire, Preston PR1 2HE, UK

3 Institute of Neurosurgery, Università Cattolica del Sacro Cuore, Rome, Italy

4 Istituto Superiore di Sanità, Department of Hematology, Oncology and Molecular Medicine, Rome, Italy

*Corresponding Author: Vittorio de Franciscis, IEOS-CNR, Via T. de Amicis 95, 80145 Naples Italy, e-mail: defranci@unina.it

Running title: Selective therapeutic targeting of glioblastoma stem-like cells 


\begin{abstract}
A minor population of glioblastoma stem-like cells (GSCs) has been implicated in the relapse and resistance of glioblastoma to therapeutic treatments. Based on knowledge of the involvement of multiple microRNAs in GSC propagation, we designed a combinational approach to target the GSC population with multiple miRNA-based therapeutics. As carriers for the targeted delivery we took advantage of two aptamers that bind to, and inhibit, the receptor tyrosine kinases, Axl and PDGFR $\beta$. We showed that the aptamer conjugates are transported through an in vitro blood-brain barrier (BBB) model. Furthermore, combining miR-137 and antimiR-10b synergizes with the receptor inhibitory function of aptamer carriers and prevents GSC expansion. Results highlighted the potential of combining multifunctional RNA-based therapeutics for selective targeting of GSCs and offer a proof of principle strategy to potentially fulfill the still unmet need for effective and safe treatment of glioma.
\end{abstract}

Keywords: aptamer, cancer stem cells, glioblastoma, microRNA, targeted delivery 


\section{Introduction}

Glioblastoma (GBM) is the most frequent and aggressive primary brain tumor in adults, classified as grade IV (World Health Organization) [1]. Treatments for GBM patients consist of tumor resection, radiotherapy and chemotherapy [2]. However, despite advances in surgical and medical neuro-oncology, prognosis for GBM patients remains dismal, with a median survival of 14-15 months [3]. Subtypes of GBM have been classified into neural, proneural, classical and mesenchymal showing typical protein-coding gene mutations as well as aberrantly expressed subsets of RNAs [4-6].

A minor cell population, constituted of multipotent tumor-initiating stem-like cells (GSCs), has been recently implicated in GBM recurrence and resistance to conventional treatments [7-9]. Indeed, GSCs are endowed with several growth properties typical of neural stem cells, including self-renewal by symmetric and asymmetric division dependent upon several factors, including the functional interplay with surrounding tumor and tumor-associated cells [10]. In comparison to the highly proliferating cells from the tumor bulk, this rare quiescent cell population is able to reconstitute the tumor mass and spread into the brain. The selective targeting of this functionally distinct, highly tumorigenic and invasive cell population is therefore considered critical for the success of therapeutic strategy to reduce tumor burden in GBM. The need for precise and safe targeted cell delivery and the lack of effective drugs to eradicate GSCs has revealed this as a challenging objective. Further, the presence of the tight cerebrovascular barrier hampers the passage of large macromolecules to target the brain tumor cells [11].

MicroRNAs (miRNAs) are short naturally occurring non-coding RNAs that prevent protein expression by forming imperfect base pairing with sequences in the $3^{\prime}$ untranslated region of target genes [12]. MiRNAs act in combination to regulate gene expression and coordinately modulate key biological processes, including cell cycle, apoptosis, differentiation, motility, epithelial-mesenchymal transition and angiogenesis, however these processes are often deregulated in tumors. As such, miRNAs are considered molecules of clinical potential in cancer treatment either as therapeutic targets or therapeutics [13].

In GSCs the combined deregulation of a characteristic pattern of miRNA expression has been described by several groups where elevated expression of a subset of miRNAs, including both miR-10a and miR-10b and downregulation of tumor suppressor miRNAs, such as miR-124, miR-9, miR-137, miR-135 have been observed in several cancer types [14-18]. Despite the potential held by miRNA-based therapeutics, major hurdles to the translation to clinic include the lack of safe and effective carriers able to penetrate and to accumulate into tumor selectively 
targeting the GSC population. In the past decade, much attention has been focused on RNAbased aptamers that are emerging as safe delivery vehicles for targeted cancer therapeutics [19]. In addition to inhibitory function of selected targets, aptamers raised against transmembrane receptors rapidly internalize into the target cell through recycling of the bound receptor [20]. So far, various therapeutic cargoes have been successfully conjugated to aptamers, including anti-cancer drugs, toxins, radionuclides, and short therapeutic RNAs (siRNAs and miRNAs) [19, 21, 22]. The main advantages for aptamer use in vivo as cell targeting moieties, include the extraordinary high binding specificity, long-term stability, little to no immunogenicity and off target toxicity [23]. Further, because of their small size, aptamers easily penetrate tumor tissues and tumor-derived spheres [24].

In our laboratory we have previously identified and characterized two internalizing RNA aptamers, GL21.T and Gint4.T, that are able to bind at high affinity and inhibit the intracellular signaling of tyrosine kinase receptors (RTKs) Axl and the platelet derived growth factor receptor $\beta$ (PDGFR $\beta$ ) respectively $[25,26]$. Axl and PDGFR $\beta$ overexpression has been reported in several human cancers and associated with invasiveness and therapeutic resistance [27,28]. Expression of PDGFR- $\beta$ is also high in atherosclerotic plaques and following vascular injury but low in normal human arteries [29, 30]. As we have recently shown, both GL21.T and Gint4.T may act as carriers for the selective delivery of short miRNA mimics and single chain miRNA antagonists, antimiR, into receptor expressing tumor cells both in vitro and in vivo. Furthermore, we have demonstrated the combinatorial potential of conjugating the GL21.T aptamer to a miRNA (or antimiR) molecule that combines the clinical benefits of both moieties [21, 22].

Here we sought to develop a mechanism-based combinational approach to target and eradicate the GSC population. To this end, we investigated the combination of miR-137 and the antagonist of the miR-10b. The miR-137 is a brain-enriched miRNA that acts as an oncosuppressor in several tumors, including GBMs, leading to decreased cell proliferation and invasion, [15] whereas miR-10b acts as an oncomiR and is highly expressed in GBMs and low expression is observed in normal human brain [18]. Antagonizing miR-10b expression has been shown to reduce glioma cell growth by cell cycle arrest and apoptosis [18].

Here we used the GL21.T and Gint4.T aptamers as carriers for miR-137 and antimiR-10b molecules to target in combination the stem-like glioma cancer cells (Axl+ / PDGFR + ) in a receptor-dependent manner. The resulting multifunctional "smart drugs" combines the inhibitory functions of the two aptamers on their target receptors with the gene regulatory function of the miRNA/antimiR cargoes, and also increases selective targeting for cells expressing both receptors. 
Results demonstrate that upon targeting the GSC population with aptamer-miRNA/antimiR conjugates, these cells differentiate, losing their ability to propagate and to sustain non-adherent growth as spheroids. 


\section{Materials and Methods}

Aptamers and conjugates

GL21.T: 5' AUGAUCAAUCGCCUCAAUUCGACAGGAGGCUCAC 3';

GL21.T stick: 5' AUGAUCAAUCGCCUCAAUUCGACAGGAGGCUCACXXXXGUACAUUC UAGAUAGCC 3';

Gint4.T: 5' UGUCGUGGGGCAUCGAGUAAAUGCAAUUCGACA 3';

Gint4.T stick: 5' UGUCGUGGGGCAUCGAGUAAAUGCAAUUCGACAXXXXGUACAUUC UAGAUAGCC 3';

Control aptamer (indicated as Ctrl aptamer):

5' UUCGUACCGGGUAGGUUGGCUUGCACAUAGAACGUGUCA 3';

Control aptamer .stick (Ctrl aptamer used in the control conjugates):

5' GCCGCUAGAACCUUCUAAGCGAAUACAUUACCGCXXXXGUACAUUC UAGAUAGCC 3';

antimiR-10b stick (indicated as antimiR-10b):

5'CACAAAUUCGGUUCUACAGGGUAGGCUAUCUAGAAUGUAC 3';

miR137 Passenger stick:

5' GGUGACGGGUAUUCUUGGGUGGAUAAUAGGCUAUCUAGAAUGUAC 3'

miR137 guide: 5' UGUUAUUGCUUAAGAAUACGCGUAGUCUU 3'

FAM labelled aptamer or aptamer stick are labelled at 5'. All RNA sequences were synthesized by tebu-bio srl (Magenta, Milan, Italy) and contained 2'-F-Py. Stick sequences (underlined) consist of 2'-F-Py and 2'-oxygen-methyl purines. The italic $X$ indicates a covalent spacer, a three-carbon linker $((\mathrm{CH} 2) 3)$. Before each treatment, aptamers were subjected to a short denaturation-renaturation step $\left(5 \min 85^{\circ} \mathrm{C}, 3 \mathrm{~min}\right.$ on ice, $10 \mathrm{~min}$ at $\left.37^{\circ} \mathrm{C}\right)$.

To prepare conjugates: 1) stick antimiR-10b were incubated at $95{ }^{\circ} \mathrm{C}$ for $10 \mathrm{~min}$ in annealing buffer (20 mM 2-[4-(2- hydroxyethyl)piperazin-1-yl] ethane sulfonic acid (HEPES) pH 7.5, 150 $\mathrm{mM} \mathrm{NaCl}, 2 \mathrm{mM} \mathrm{CaCl}$ ) or miR-137 passenger and guide were annealed by incubating in annealing buffer at $95^{\circ} \mathrm{C}$ for $10 \mathrm{~min}$, at $55^{\circ} \mathrm{C}$ for $10 \mathrm{~min}$ and then at $37^{\circ} \mathrm{C}$ for $20 \mathrm{~min}$; 2) stick aptamers were refolded $\left(5 \mathrm{~min} 85^{\circ} \mathrm{C}, 3 \mathrm{~min}\right.$ on ice, $10 \mathrm{~min}$ at $37^{\circ} \mathrm{C}$ ), 3) equal amounts (ratio $1: 1)$ of stick aptamer and antimiR-stick/annealed passenger-guide were then annealed by incubating together at $37^{\circ} \mathrm{C}$ for $30 \mathrm{~min}$. The annealing efficiency was evaluated on a $12 \%$ nondenaturing polyacrylamide gel.

Cells and transfection 
Human glioma U87MG cells were provide from ATCC (American Type Culture Collection, Manassas, VA) and grown in Dulbecco's modified Eagle's medium (DMEM, Sigma Aldrich, St Louis, MO, USA) supplemented with $10 \%$ fetal bovine serum (FBS) (Sigma Aldrich, St Louis, MO, USA). To obtain U87MG-derived tumorspheres, cells were grown at low density (2000 cells/ml) in serum-free DMEM-F12 (Sigma Aldrich, St Louis, MO, USA) supplemented with B27, $20 \mathrm{ng} / \mathrm{ml}$ EGF and $20 \mathrm{ng} / \mathrm{ml} \mathrm{bFGF}$ (Life technologies Milan Italy) for 10-15 days allowing the appearance of floating spheres. Glioblastoma stem-like cells were provided by Dr Lucia RicciVitiani and cultured in a serum-free medium supplemented with $20 \mathrm{ng} / \mathrm{ml}$ EGF and $10 \mathrm{ng} / \mathrm{ml}$ bFGF (Life technologies Milan Italy) as reported [31].

For treatments, tumorspheres were collected by gentle centrifugation $(800 \mathrm{rpm})$, dissociated with $0.25 \%$ trypsin for $5 \mathrm{~min}$ and treated as indicated with molecular conjugates or aptamers in supplemented serum-free appropriate medium. Treatments were renewed by adding aptamer/conjugates $(200 \mathrm{nmol} / \mathrm{l})$ three times a week, as indicated. Spheroids were then collected and analyzed, or (for data in Fig. 3E) dissociated and plated at same density in rich medium without treatments for further 10 days before analyses.

All transfections were performed using serum-free Opti-MEM and Lipofectamine RNAimax reagent (Invitrogen,Waltham, MA, USA) according to the manufacturer's protocol. Cells were transfected with $100 \mathrm{nmol} / \mathrm{l}$ (otherwise indicated) of: Anti-miR ${ }^{\mathrm{TM}}$ miRNA Inhibitor Negative Control (ctrl antimiR); Pre-miR ${ }^{\mathrm{TM}}$ miRNA Precursor Negative control and Pre-miR ${ }^{\mathrm{TM}}$ miRNA Precursor miR-137 (miR-137) or with antimiR-10b (Ambion, Waltham, MA, USA).

\section{Immunoblot analysis}

Total cell lysates were prepared in JS buffer ( $50 \mathrm{mM}$ Hepes (pH 7.5), $150 \mathrm{mM} \mathrm{NaCl}, 1 \%$ glycerol, $1 \%$ Triton X-100,1.5mMMgCl2, 5mMEGTA, $1 \mathrm{mMNa} 3 \mathrm{VO} 4$, protease inhibitors) and then boiled in sodium dodecyl sulfate/ $\beta$-mercaptoethanol sample buffer. Proteins were separated by electrophoresis and then blotted onto polyvinylidene difluoride membranes (Millipore, Billerica, MA, USA) by an electrophoretic transfer. After blocking with $5 \%$ dried milk in Tris-buffered saline (TBS) containing $0.1 \%$ Tween-20, membranes were incubated at $4{ }^{\circ} \mathrm{C}$ overnight with the following primary antibodies: anti-PDGFR- $\beta$ (Cell Signalling Technology, Inc., Danvers, MA, USA); anti-Bim (Cell Signalling Technology, Inc., Danvers, MA, USA); anti-Sox-2 (Abnova, Taipei City, Taiwan); anti-AxI (R\&D Systems, Minneapolis, MN, USA); anti-Nanog (Santa Cruz Biotechnology, Stockton, CA, USA); anti-GLIPR-1 (Abcam, Cambridge, UK); antiSlug (Santa Cruz Biotechnology, Stockton, CA, USA); anti-a-tubulin (Santa Cruz Biotechnology, 
Stockton, CA, USA); anti $\beta$-actin (Sigma Aldrich, St Louis, MO, USA). Blots were quantified with Image $\mathrm{J}$ software.

\section{Cell viability assays}

Dissociated tumorspheres were seeded in 96 -well plates $\left(2 \times 10^{3}\right.$ cells/well $)$ and left untreated, transfected with indicated miRs/antimiRs or treated with aptamers or conjugated, as indicated. Cell viability was assessed by CellTiter 96 Proliferation Assay (Promega, Madison, WI) after gentle pipetting.

\section{Flow cytometry analysis}

For cell cycle analyses, dissociated U87MG-derived tumorspheres or U87MG were left untreated or treated with aptamers or chimeras (400 nmol/l final concentration) for 10 days. Treatments were renewed by adding aptamer/conjugates $(200 \mathrm{nmol} / \mathrm{l})$ three times a week. Cells were centrifuged, dissociated, washed with $1 \mathrm{ml}$ of cold $1 \mathrm{X}$ PBS and then fixed with ethanol $70 \%$. Samples were then washed twice with $1 \mathrm{ml}$ of cold PBS to completely remove ethanol. Cells pellet was then suspended in $400 \mu$ of PBS with RNase A and Propidium lodide (PI, Sigma, St Louis, MO) to a final concentration of $50 \mu \mathrm{g} / \mathrm{ml}$. Tubes were incubated for 20 minutes at $37^{\circ} \mathrm{C}$. Cell cycle were analyzed with BD Accuri C6 (BD Biosciences, Franklin Lakes, New Jersey, USA).

For the Annexin V-FITC apoptosis detection assay, dissociated U87MG-derived tumorspheres were left untreated or treated with aptamers or chimeras (400 nmol/l final concentration) for 3 days. Cells were centrifuged for 5 minutes and washed twice with $1 \mathrm{ml}$ of cold $1 \mathrm{X}$ PBS and then suspended in $1 \mathrm{X}$ binding buffer at a concentration of $1 \times 10^{6}$ cells $/ \mathrm{ml}$. Annexin V-FITC and PI (Novus Biologicals, Littleton, CO, USA) were added at $100 \mu \mathrm{l}$ of each sample according to the manufacturer's recommendations (5 $\mu \mathrm{L}$ of Annexin V-FITC $+\mathrm{PI}$ ). Samples were then incubated for 20 minutes at RT in the dark. $400 \mu$ of $1 \mathrm{X}$ binding buffer was added to each sample before flow cytometry analysis with BD Accuri C6 (BD Biosciences, Franklin Lakes, New Jersey, USA). $A x l$ and PDGF R $\beta$ expression of GSCs were detected by anti-human Axl and PDGF R $\beta$ goat IgG antibodies (R\&D Systems, Milan, Italy). Alexa Fluor 647 donkey anti-goat IgG (Invitrogen, Milan, Italy) was used as secondary antibody. Viable cells were identified using 7-amino actinomycin D (7AAD; Sigma Aldrich, St. Louis, MO). Cells were analyzed with FACSCanto flow cytometer (Becton Dickinson, Milan, Italy).

\section{Immunofluorescence}


To assess Axl and PDFGR $\beta$ expression, U87MG-derived tumorspheres were seeded on poly-LLysine coated glass coverslips and incubated with anti-Axl or anti- PDFGR $\beta$ antibodies (R\&D System, Minneapolis, MN) for $30 \mathrm{~min}$ at $37{ }^{\circ} \mathrm{C}$ prior to fixation. Cells were then fixed with paraformaldehyde $4 \%$ in PBS for 10 minutes and incubated at $37{ }^{\circ} \mathrm{C}$ for 30 minutes with Alexa568 secondary antibody (Invitrogen, Waltham, MA, USA). Coverslips were then mounted on microscope slides with prolong gold antifade reagent with DAPI (Invitrogen, Waltham, MA, USA) and visualized by confocal microscopy. Images were obtained using a Zeiss 510 LSM confocal microscope with a $40 x$ oil objective.

\section{Transwell migration assay}

Dissociated tumorspheres were counted and $1.4 \times 10^{5}$ cells/point were treated $(400 \mathrm{nmol} / \mathrm{l}$ final concentration) or transfected as indicated. Following $24 \mathrm{~h}, 1 \times 10^{5}$ cells were plated into the upper chamber of a 24-well transwell (Corning Incorporate, Corning, NY) in serum free DMEM and exposed to $10 \%$ FBS as inducers of migration $(0.6 \mathrm{ml}$, lower chamber) for additional $24 \mathrm{~h}$. Migrated cells were visualized by staining with $0.1 \%$ crystal violet in $25 \%$ methanol and photographed with Leica Application Suite. Percentage of migrated cells was evaluated by eluting crystal violet with $1 \%$ sodium dodecyl sulfate and reading the absorbance at $594 \mathrm{~nm}$ wavelength.

\section{Tumorsphere formation assay}

Tumorsphere formation assay was performed in 96 well plate in duplicate. Dissociated tumorspheres were counted and 500 cells/well were maintained in stem medium in the absence or in presence of aptamers or chimeras at $400 \mathrm{nmol} / \mathrm{l}$ concentration. Treatments were renewed by adding aptamer/conjugates $(200 \mathrm{nmol} / \mathrm{l})$ three times a week. Following 10 days, wells were photographed with Leica Application Suite and spheres with a diameter greater than $25 \mu \mathrm{m}$ were counted.

\section{Chimeras stability in human serum}

GL21.T-137, GL21.T-10b, Gint4.T-137 and Gint4.T-10b chimeras were incubated at $4 \mu \mathrm{M}$ in $80 \%$ human serum from $1 \mathrm{~h}$ to 7 days. Type AB Human Serum provided by Euroclone (Cat. ECS0219D) was used. At each time point $8 \mu \mathrm{l}(32 \mathrm{pmol}$ RNA) was withdrawn and incubated for $2 \mathrm{~h}$ at $37^{\circ} \mathrm{C}$ with $1 \mu \mathrm{l}$ of proteinase $\mathrm{K}$ solution $(600 \mathrm{mAU} / \mathrm{ml})$ in order to remove serum proteins that interfere with electrophoretic migration. Following proteinase $\mathrm{K}$ treatment, $9 \mu \mathrm{l} 1 \times \mathrm{TBE}$ and $3 \mu \mathrm{l}$ gel loading buffer (Invitrogen, Waltham, MA, USA) were added to samples that were then 
stored at $-80{ }^{\circ} \mathrm{C}$. All time point samples were separated by electrophoresis into $10 \%$ nondenaturing polyacrylamide gel. The gel was stained with ethidium bromide and visualized by UV exposure.

Reverse transcription quantitative polymerase chain reaction (RT-qPCR)

For Real-time PCR, RNAs were extracted with TRiZol (Invitrogen, Waltham, MA, USA). To assess miRNA levels $500 \mathrm{ng}$ of total RNA was reverse transcribed with miScript Reverse Transcription kit (Qiagen, Milan, Italy) according to the manufacturer's protocol. Amplification was performed by using the miScript-SYBR Green PCR Kit and specific miScript Primer Assay (Qiagen, Milan, Italy). U6 RNA was used as a housekeeping control gene.

To evaluate PDGFR- $\beta$, Nanog, Sox-2, Shh, Oct-4, GFAP and TUBB-3 mRNA levels, $1 \mu \mathrm{g}$ of total RNA was reverse transcribed with iScript cDNA Synthesis Kit and amplified with IQ-SYBR Green supermix (Bio-Rad, Hercules, CA, USA). $\beta$-actin was used as a housekeeping control gene. The specific primers used were: GFAP: fw 5' CTGCGGCTCGATCAACTCA 3', rev. 5' TCCAGCGACTCAATCTTCCTC 3'; TUBB-3: fw 5' GAGCGGATCAGCGTCTACTAC 3', rev. 5' CCCCACTCTGACCAAAGATGAA 3'; Nanog: fw 5' CTAAGAGGTGGCAGAAAAACA 3', rev 5' CTGGTGGTAGGAAGAGTAAAGG 3; Sox-2: fw 5'GCACATGAACGGCTGGAGCAAGC 3', rev. 5' TGCTGCGAGTAGGACATGCTGTAGG 3'; Shh: fw 5'TCGGTGAAAGCAGAGAAC 3', rev 5' AGgAAAGTGAGGAAGTCG 3'; PDGFRß : fw 5' GCTCACACTGACCAACCTCA 3', rev. 5' GGTGGGATCTGGCACAAAGA 3'; Oct-4: fw 5' CGAAAGAGAAAGCGAACCAG 3', rev. 5' GCCGGTTACAGAACCACACT 3'; $\beta$-actin: fw: 5' CAAGAGATGGCCACGGCTGCT 3'; rev.: 5' TCCTTCTGCATCCTGTCGGCA 3'.

TaqMan Array Human Molecular Mechanisms of Cancer (Applied Biosystems, Foster City, CA, USA) were performed following manufacturer's instructions by using a TaqMan Universal PCR Master Mix (Applied Biosystems, Foster City, CA, USA) and 50ng cDNA as input. Three endogenous control gene (GAPDH, HPRT1, GUSB) were used as housekeeping control genes. Relative mRNA and miRNA quantization was performed by using the $\triangle \triangle \mathrm{Ct}$ method applying the equation $2^{-\triangle \Delta C t}$.

For miRNA absolute quantitation, RNAs were recovered in TRIzol containing $0.5 \mathrm{pmol} / \mathrm{ml}$ of CL4 aptamer (CL4: 5' GCCUUAGUAACGUGCUUUGAUGUCGAUUCGACAGGAGGC 3') used as a reference control. Amplification were performed with $50 \mathrm{ng}$ of RNA retrotranscribed with miScript Reverse Transcription kit. Standard curves with purified miR-137 and miR-10b (from Eurofins Genomics, Milan, Italy) were performed to quantity samples and number of copies 
were calculated (number of copies $=\left(\mathrm{ng} * 6.022 \times 10^{23}\right) /\left(\right.$ length $\left.\left.* 1 \times 10^{9 *} 650\right)\right)$. Data were normalized to the CL4 reference control.

In vitro blood brain barrier model

The all human in vitro tri-culture cell model of the BBB was set up using short-term cultures of endothelial cells (HBMEC, SC1000), astrocytes (HA, SC1800) and pericytes (HBVP, SC1200) (ScienCell Research Laboratories, CA, USA), based on a previously published method (Kumar et al., 2014). The transendothelial electrical resistance (TEER) of the BBB was measured using the EVOM-2 (Merck Millipore, Oxford, UK) on the insert for each of the models every day for 7 days, to ensure barrier formation. On day 8 , the permeability of the FAM-aptamers and chimeras were tested by seeding the aptamers in the apical side of the tri-culture at a final concentration of $2.5 \mu \mathrm{M}$. At set time intervals $(0.25,0.5,1,1.5,2,4,6,8,24 \mathrm{~h})$, the TEER was measured and media from the basolateral side of the transwell insert was sampled media and fluorescent read on a Tecan GENios Pro® plate reader (Tecan UK Ltd., Theale, UK) excitation and emission wavelength $492 \mathrm{~nm}$ and $518 \mathrm{~nm}$ respectively. The apparent permeability of each aptamer was calculated using the formula in Equation 1 (Artursson, 1990). All data was obtained from three experimental replicates.

$$
P_{a p p}=\frac{V}{A \times C_{0}} \times \frac{d Q}{d t}
$$

\section{(Equation 1)}

Where: $V=$ Volume of basolateral compartment $\left(0.5 \mathrm{~cm}^{3}\right) ; A=$ surface area of the polycarbonate membrane $\left(0.3 \mathrm{~cm}^{2}\right) \mathrm{C}_{0}=$ Final concentration of the FAM-aptamer in the apical side $(2.5 \mu \mathrm{M}) \mathrm{dQ}$ Concentration of FAM-aptamer passing across the cell layer to basolateral side $(\mu \mathrm{M}) \mathrm{dt}=\mathrm{Time}$ (min).

\section{Statistical analyses}

Statistical analyses were performed by one-way Anova with GraphPad Prism v.6.0 as indicated. 


\section{RESULTS}

\section{miR-137 and anti-miR-10b combination in U87MG-derived stem cells}

We first used tumor-spheres from U87MG cancer cells to determine whether interfering with multiple miRNA expression would revert the stem-like phenotype. As shown in Supplementary Fig. 1, upon induction of tumorsphere growth by serum-free culturing conditions the U87MG cells express a stem-like phenotype associated with the down regulation of miR-137 levels and up-regulation of miR-10b levels. In U87MG tumorspheres, we restored the relative expression of these two miRNAs by transfecting the miR-137 mimic and the miR-10b antagonist (antimiR10b), either separately or in combination. As shown in Fig. 1A, both the miR-137 mimic and the antimiR-10b alone and in double transfections retain functional activity as determined by the simultaneous downregulation of the miR-137 validated target, glioma pathogenesis-related protein 1 (GLIPR-1) [15], and up-regulation of the miR-10b validated target, BH3-only protein, (Bim) [18]. Further, both molecules synergize to inhibit cell migration (Fig. 1B) and cell viability (Fig. 1C) of transfected cells, ultimately inhibiting the self-renewal potential of U87MG cells as indicated by the reduced number of spheres in double-treated cells as compared to controls (Fig. 1D). Results indicate that these two miRNAs synergize to regulate survival and selfrenewal of U87MG spheres.

\section{Anti-Axl and anti-PDGFR $\beta$ aptamers as functional carriers for miRNA delivery in GSCs}

As previously shown, the GL21.T and Gint4.T aptamers target and inhibit the Axl and the PDGFR $\beta$ receptors respectively $[25,26]$. While both $A x l$ and the PDGFR $\beta$ receptors are expressed in U87MG, only PDGFR $\beta$ expression is strongly enhanced in tumor-spheres, compared to 2D cultured cells (Supplementary Fig. 1A). Therefore, by adopting a stick-end approach for the non-covalent conjugation, we took advantage of GL21.T and Gint4.T as carriers for the receptor-dependent delivery of miR-137 and of antimiR-10b into tumor-derived spheres. We first designed the conjugates in the four possible configurations with the GL21.T (anti-Axl) and the Gint4.T aptamer (anti-PDGFRß) either annealed to the miR-137 or to the antimiR-10b sequences (Fig. 2A). By treating U87MG tumorspheres cells with each of the conjugates, we show that the ability to regulate the respective microRNA levels and microRNA target protein expression (Supplementary Fig. 2) are preserved. We thus treated U87MG derived tumorspheres with each of the conjugates and determined the ability to interfere with stem-like properties (Fig. 2B-F). Upon 10 days of treatment, each conjugate alone strongly reduces the number and size of spheroids. 
A
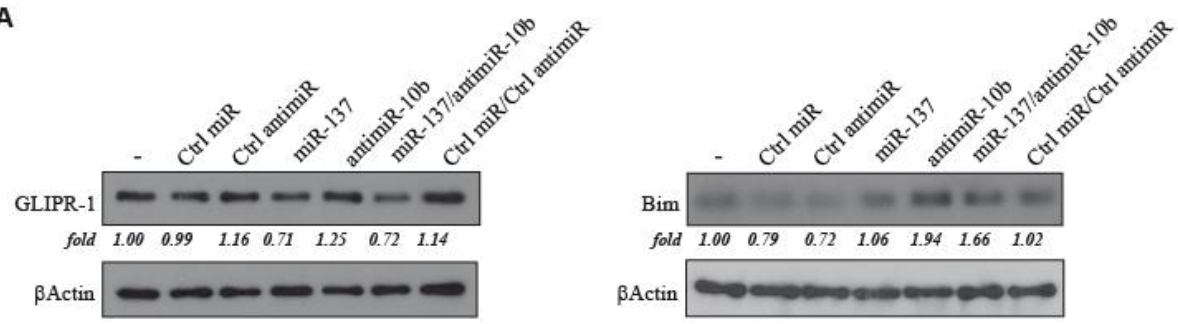

B

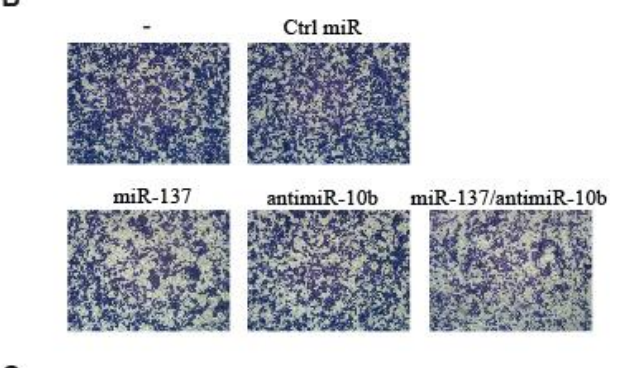

C
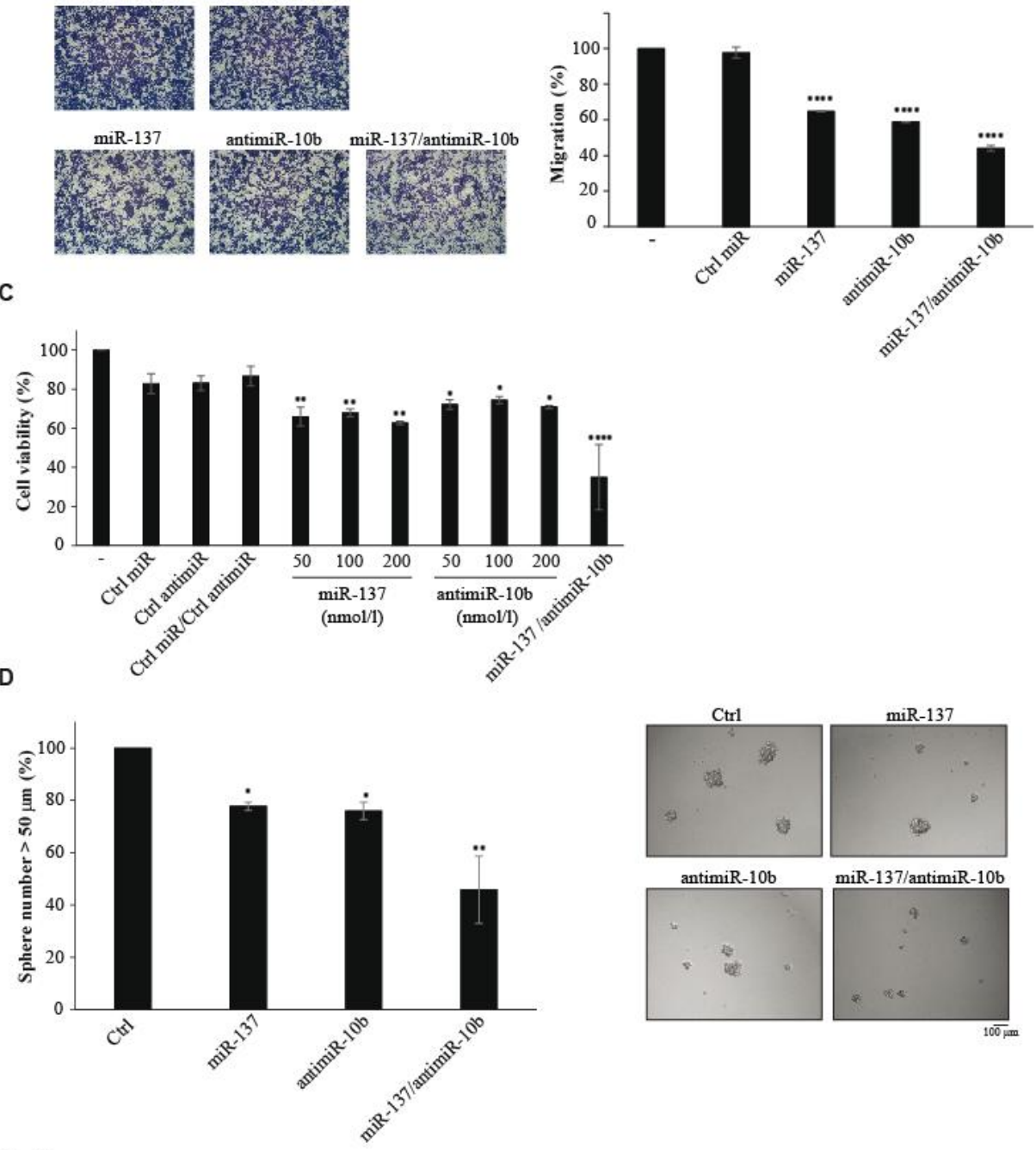

miR-137/antimiR-10b

Fig. 1 
A

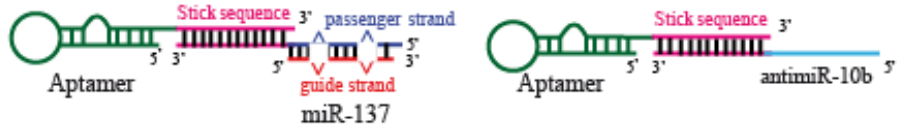

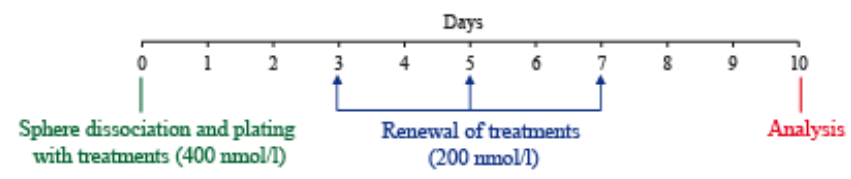

B
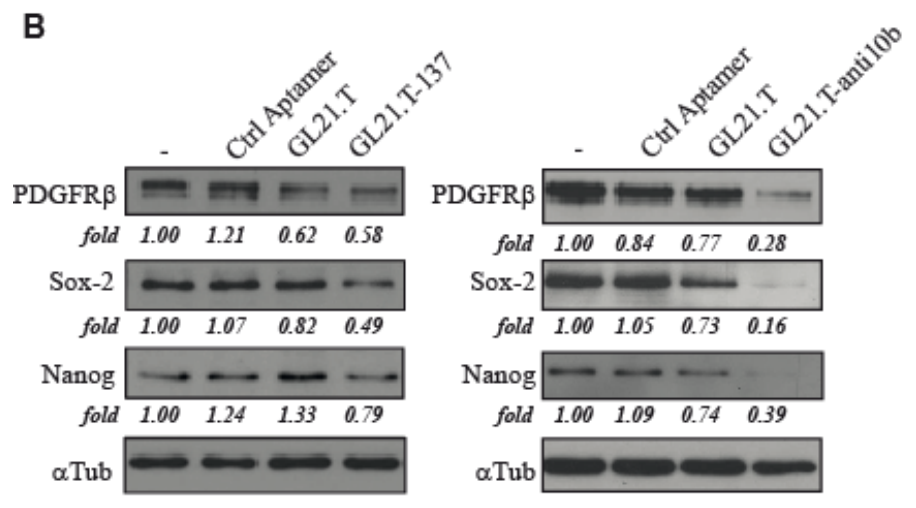

C

D

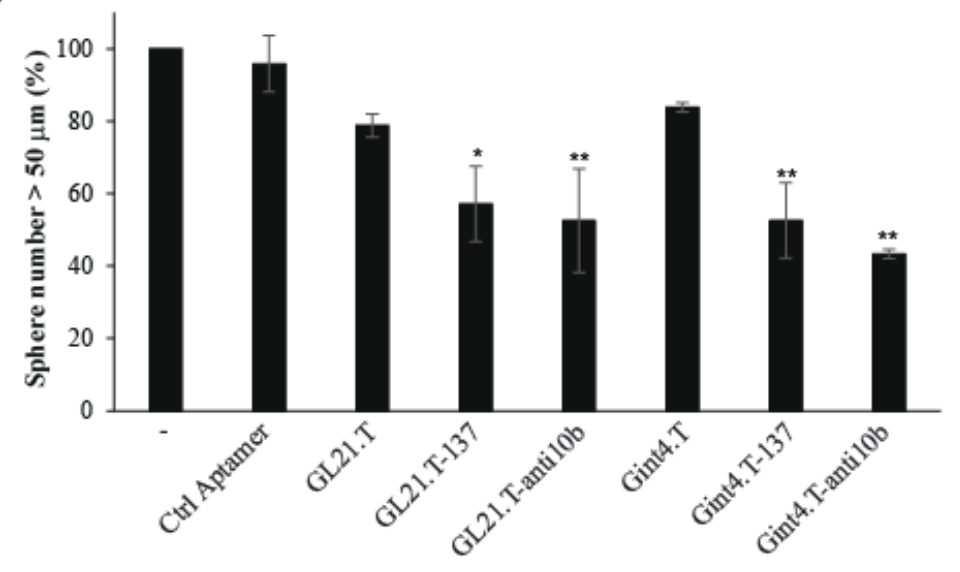

E

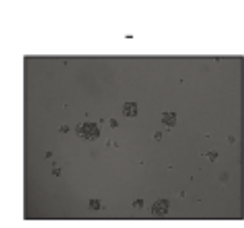

GL21.T

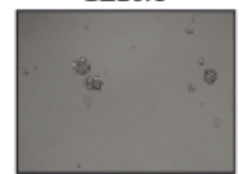

Gint4.T

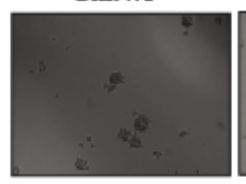

Ctrl Aptamer

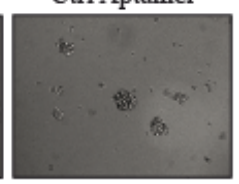

GL21.T-137

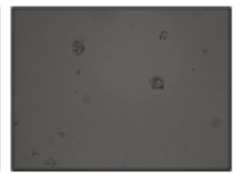

Gint4.T-137

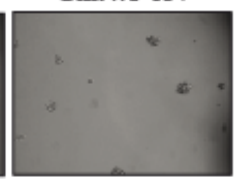

GL21.T-anti10b

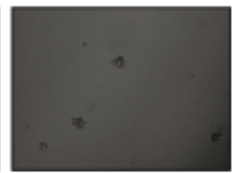

Gint4.T-anti10b

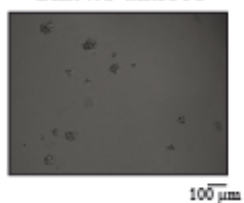

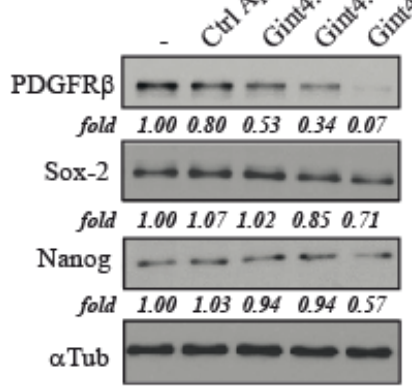

F

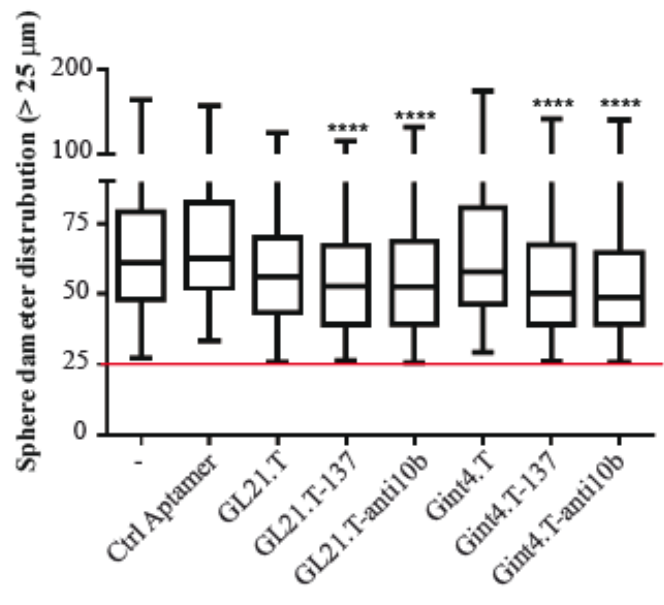

Fig. 2 


\section{The GL21.T-miR137 and Gint4.T-anti10b conjugates act in combination to affect tumorsphere formation}

As shown above, both configurations work with similar efficacy, yet, the efficiency of aptamer uptake (either of GL21.T or Gint4.T) is restrained by the amount of target receptors on the cell surface [21]. Using two different aptamers together, would thus increase the efficiency of uptake of each conjugate and would inhibit the two target receptors. On first attempt we used the GL21.T (anti-Axl) conjugated to the miR-137 and the Gint4.T aptamer (anti- PDGFR $\beta$ ) conjugated to the anti-miR-10b sequence. We determined whether combining GI21.T-miR137 and Gint4.T-anti10b to treat tumor-spheres might synergize thus antagonizing stem-like glioma cell growth. Indeed, as shown in Fig. 2B-F each aptamer conjugate interferes independently with sphere formation participating in the functional inhibition of stem cell-like phenotype. In double treatments, both conjugates acted in combination as determined by the increased intracellular miR-137 and down-regulation of the miR-10b (Supplementary Fig. 3). Treatment of U87MG-derived spheres with both conjugates strongly reduced the expression of stem-cell associated genes PDGFR , Sox-2 and Nanog and of the epithelial mesenchymal transition associated gene, SLUG (Snail2) (Fig. 3A), decreased the number (to approximately $20 \%$ of spheres $>50 \mu \mathrm{m}$ diameter) and size of sphere formation (Fig. 3B-D) indicating a strong reduction of self-renewal potential. Notably, the treatments with control aptamer conjugates as well as with a mixture containing GL21.T/Gint4.T and miR-137/antimiR-10b molecules unconjugated were unable to affect sphere formation (Supplementary Fig. 4), indicating that the functional effects were dependent upon the annealing of the miR/antimiR cargoes to the targeting aptamer. To evaluate if the surviving small tumorspheres were still able to propagate in adherent growth conditions, we dissociated the sphere aggregates from the combined treatments and from controls, plated and cultured cells in the absence of any treatment for further 10 days in 10\% FBS. As shown in Fig. 3E, the surviving spheres were constituted of poorly viable cells but were still able to propagate in 2-D cultures.

On the other hand, the clear increases in glial fibrillary acidic protein (GFAP) and $\beta$-tubulin III (TUBB3) levels in double-treated cells (Fig. 3F) indicated that the combined treatment induced the stem-like cells to differentiate acquiring an adherent-like cell phenotype similar to that of the proliferating U87MG cells. We thus determined by FACS analysis the distribution of the cell cycle phases in the treated cell populations. As shown in Fig. 4A, in contrast to adherent U87MG population, where more than $30 \%$ of cells were distributed between $S$ and G2/M (12.9 $\%$ and $20 \%$ respectively), in tumor-spheres the majority of cells were in G1 with less than $10 \%$ distributed between the $S$ and $\mathrm{G} 2 / \mathrm{M}$ as expected for a poorly replicating/quiescent cell 
population. In agreement with the expression of differentiated markers (Fig. 3F), upon treatment with the combination of conjugates the fraction of cells in G1 was clearly reduced, with a corresponding increase of cells found in the $S$ and $\mathrm{G} 2 / \mathrm{M}$ (14.1\% and $21.5 \%$ respectively); which was similar to levels observed for the adherent U87MG population. Further, the increase in cells in the pre-G1 fraction (7.2\%) indicated the presence of a fraction of cells undergoing apoptosis. This latter observation is well supported by the MTT analysis of cell viability showing that the combined treatments induced a 40 per cent reduction in cell viability (Fig. 4B) and Annexin V/PIpositive cells $(21 \%$ in the sample from combined treatments as compared to $14.5 \%$ in untreated controls) (Fig. 4C).

The intracellular processes initiated by Axl and PDGFR $\beta$ and those regulated by miR-137 and miR-10b engage multiple converging pathways in the cell, including Ras-MAPK and PI3k-Akt, therefore extensive changes in the pattern of gene expression were predicted for the treated tumorspheres. RT-qPCR array analysis of U87MG tumor-spheres showed (Fig. 4D, E) that the combined treatment drastically changed the expression of multiple genes converging on key processes such as cell cycle and apoptosis, where are ultimately responsible for GSCs differentiation and cell death. At least for the gene-set analyzed, the gene expression pattern found upon the combined treatments comes from the contribution of at least one conjugate, even though the GL21.T-miR137 seems to have by far the major impact on this set of genes. In addition, since stem-like cancer cells typically migrate in the presence of a chemoattractant stimulus, we determined whether treatment with conjugates might synergize to inhibit migration. Indeed as shown above, transfecting miR-137 synergizes with antimiR-10b to inhibit migration (Fig. 1B). Moreover, both aptamers, GL21.T and Gint4.T, have been reported to interfere with cell migration $[25,26]$. As shown in Fig. $4 \mathrm{~F}$, the combined treatment with conjugates reduces migration by approximately $60 \%$ suggesting that the pathways involved largely superpose each other. Taken together these results show that treating cells with the two conjugates in combination dramatically inhibits tumor-sphere formation and migration. 
A

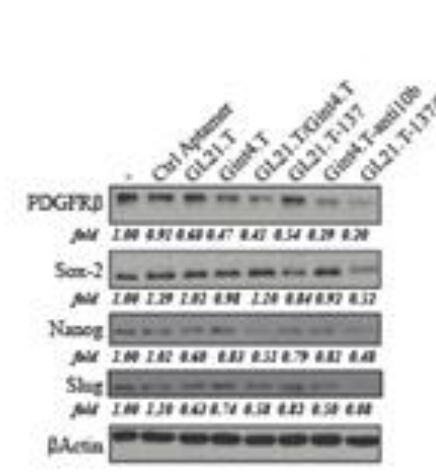

c
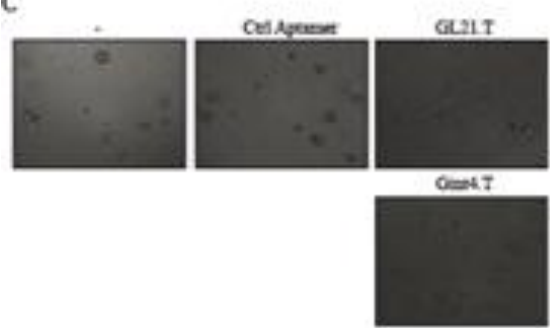

caritrcenest

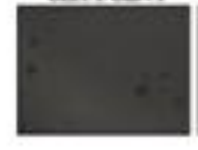

E
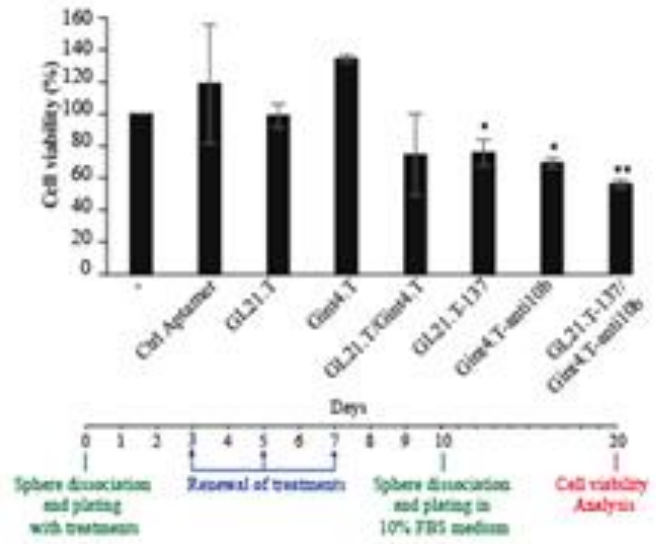

B

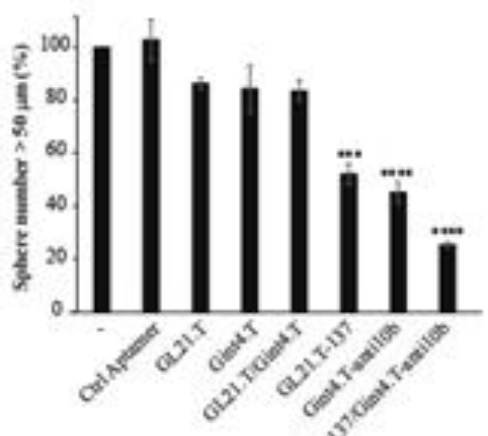

D
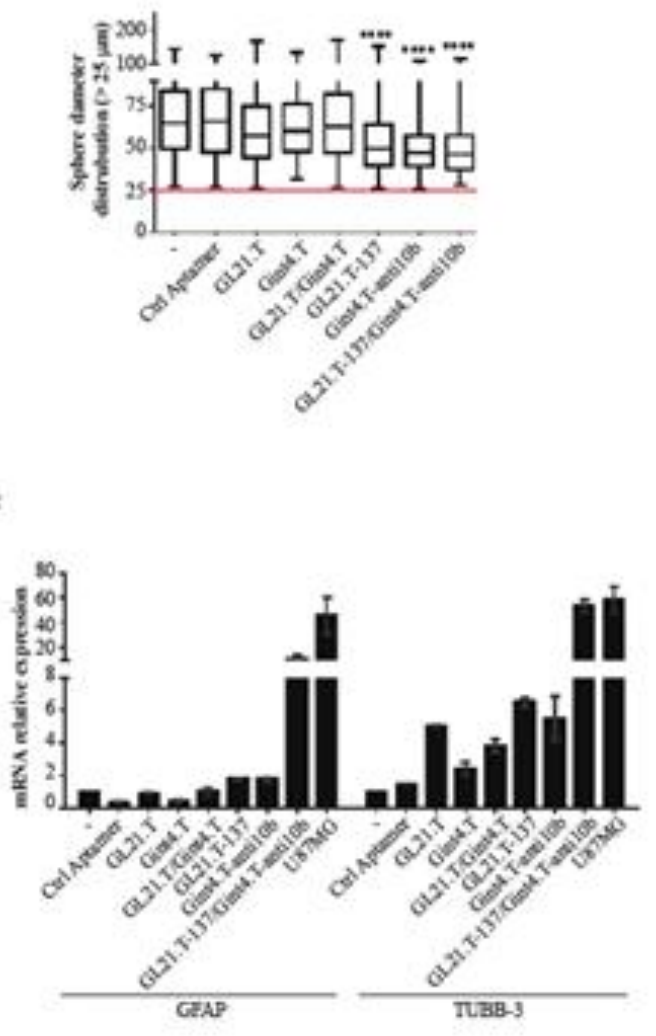

Fig. 3 
A

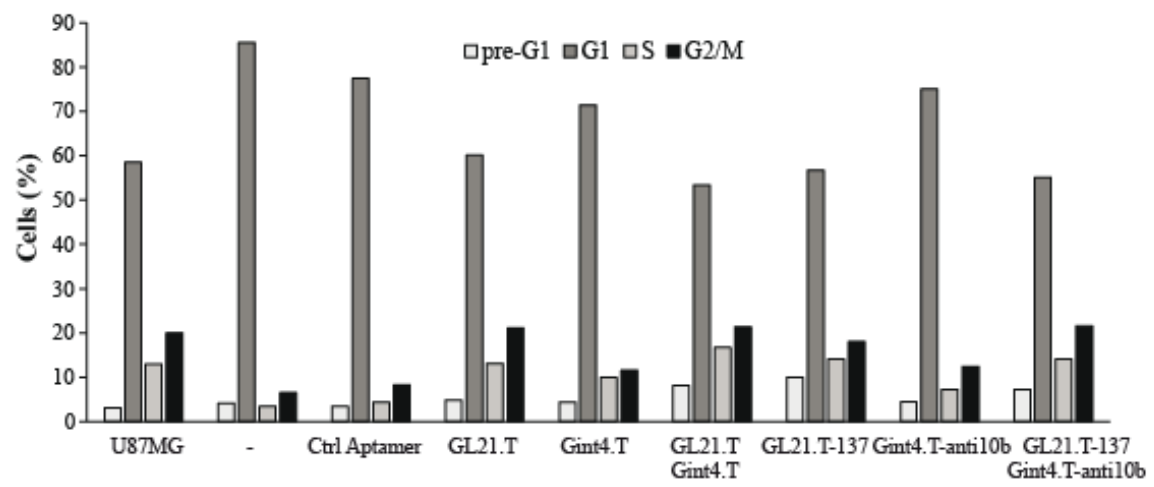

B

D

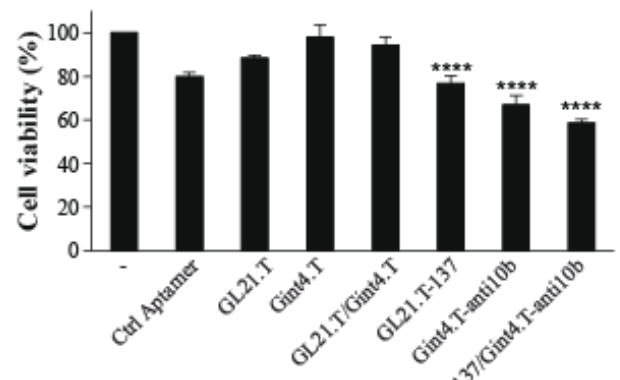

C

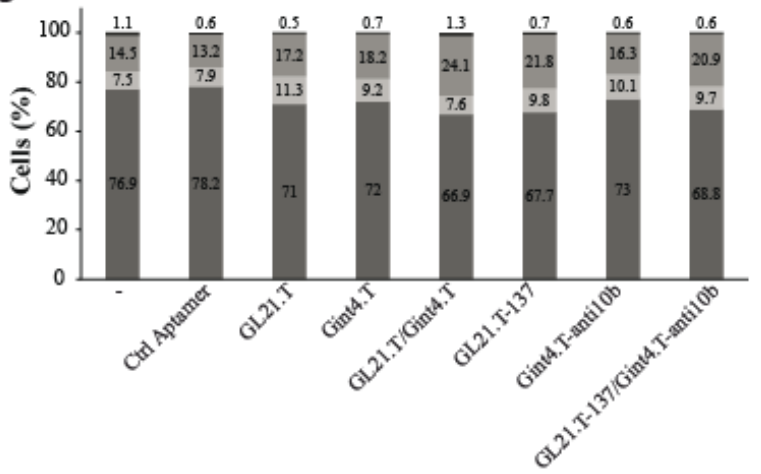

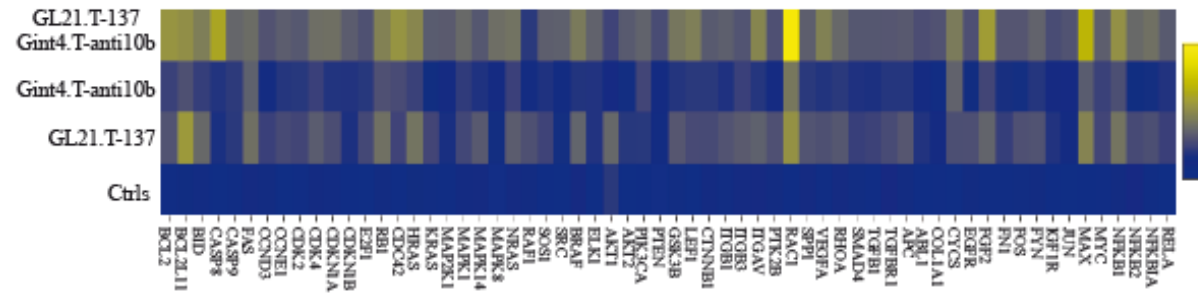

E

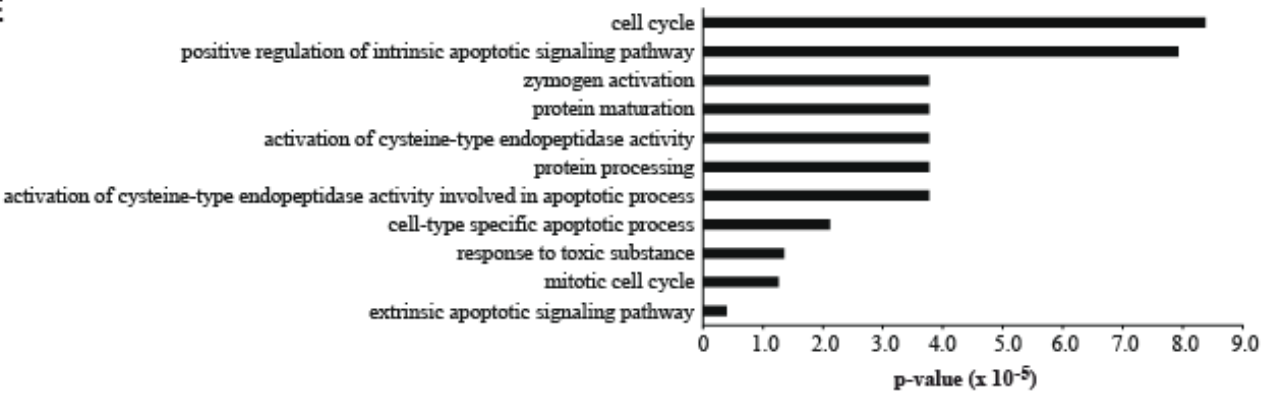

$\mathbf{F}$

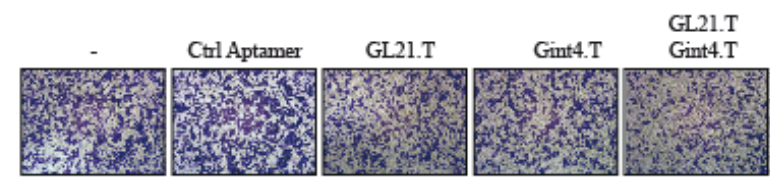

GL21 T-137

GL21.T-137 Gint4.T-anti10b Gint4.T-antil0b
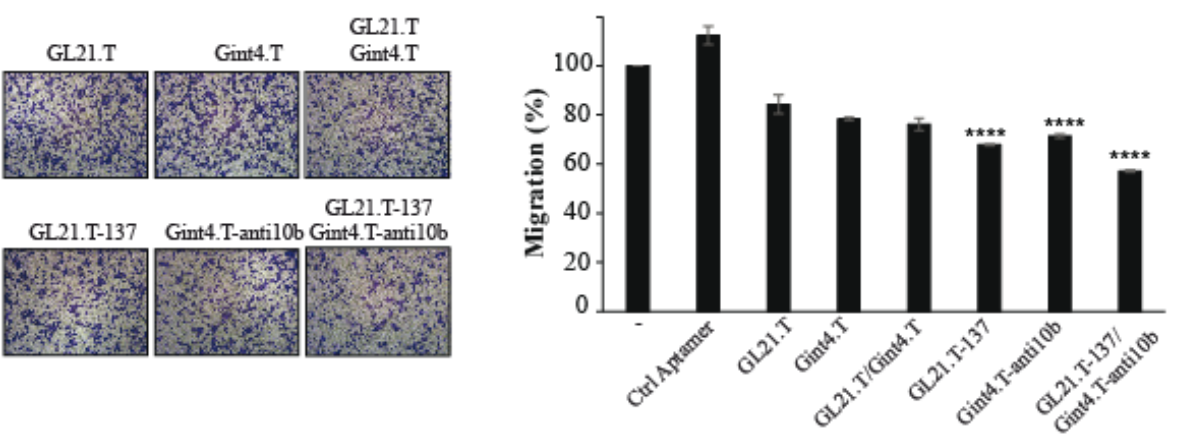

Fig. 4 


\section{The GL21.T-miR137 and Gint4.T-anti10b as inhibitors of primary GSC propagation,}

Based on the demonstration that appropriately combining aptamer-miR/antimiR conjugates inhibited the U87MG tumor-sphere cell growth we evaluated the targeting of GSCs from patient derived gliomas. From a collection of patient-derived primary human GSCs, we selected three primary cell lines displaying the more aggressive signature of GSr-like (restricted-like stem) [32]. The cell lines, named GSC\#61, GSC\#74 and GSC\#83, were derived from two classical (\#61 and \#74) and a mesenchymal (\#83) GBM subtype (according to [5]), and all expressed both Axl and PDGFR $\beta$ aptamer targets on the cell surface (Supplementary Fig. 5A, B). In contrast to U87MG, primary human GSCs were propagated in growth factors supplemented minimal medium as non-adherent tumor spheroids. The expression of miR-137 and miR-10b were evaluated by RT-qPCR as absolute amounts per microgram of total RNA. As shown in Fig. 5A, both, the miR-137 and miR-10b, were equally expressed in the three non-adherent growing cell lines with miR-10b being expressed approximately three fold more than miR-137.

The fact that the GSC\#61, GSC\#74 and GSC\#83 cells express similar absolute amounts of miRNA-137 and miRNA-10b allowed us to investigate the implication of these miRNAs in the propagation of the stem-like cell phenotype in these primary cell lines. Upon dissociation, we treated cells with conjugates either separately or in combination and left them to form clonal spheres for two weeks, and thus determined the functional inhibition of the stem-like phenotype. As previously observed with U87MG cells (Fig. 3B), each conjugate independently affected number and size of spheroids in the three cell lines. The combined action of the two conjugates synergized to drastically inhibit the ability of cells to grow as non-adherent spheroids (Fig. 5B, D and Supplementary Fig. 6 and 7) and strongly reduces cell viability (Fig. 6A-C) and migration (Fig. 6D-F), the GSC\#61 being less sensitive to treatments. Taken together these data show that the combined treatment with GL21.T-miR137 and Gint4.T-anti10b conjugates abrogate the ability of GSCs to grow as tumor spheres leading to reduced cell viability. 




B
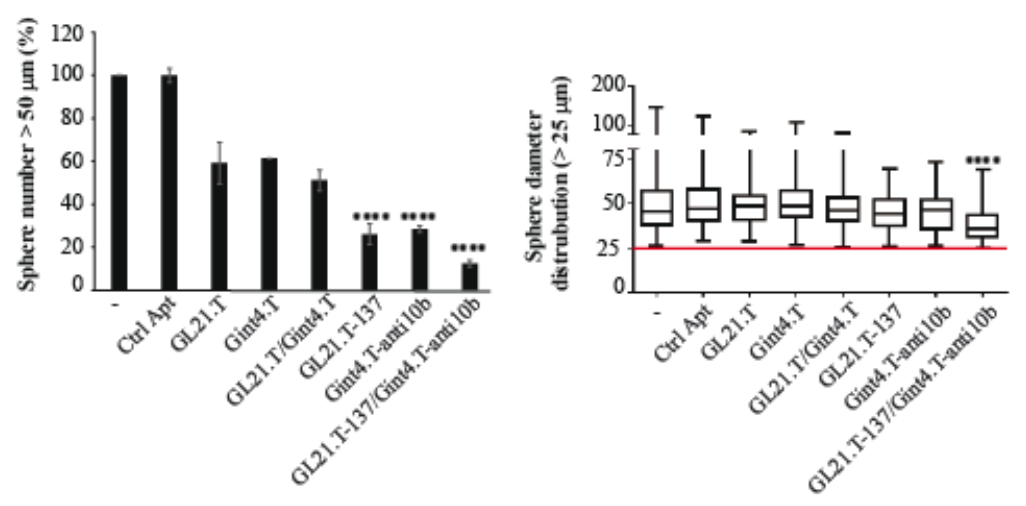

C

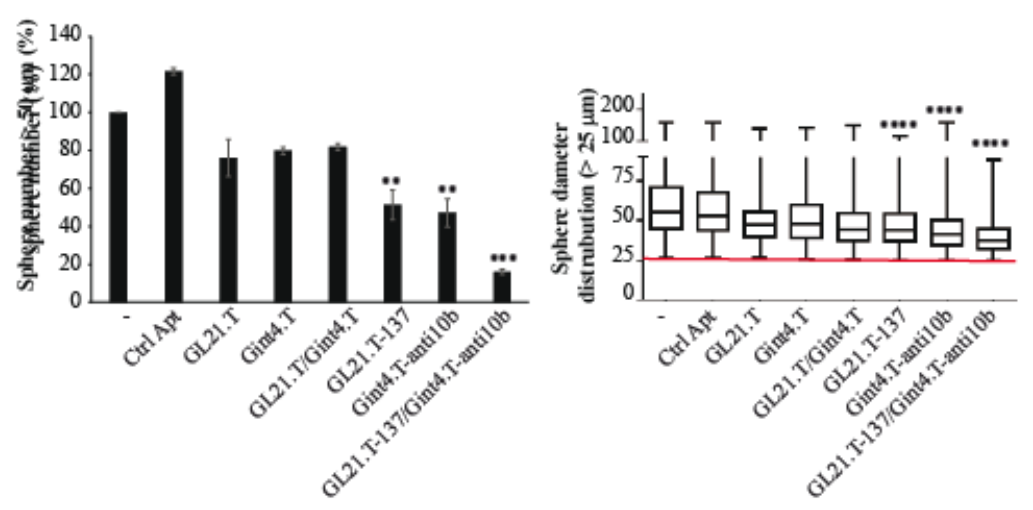

D

GSC\#61
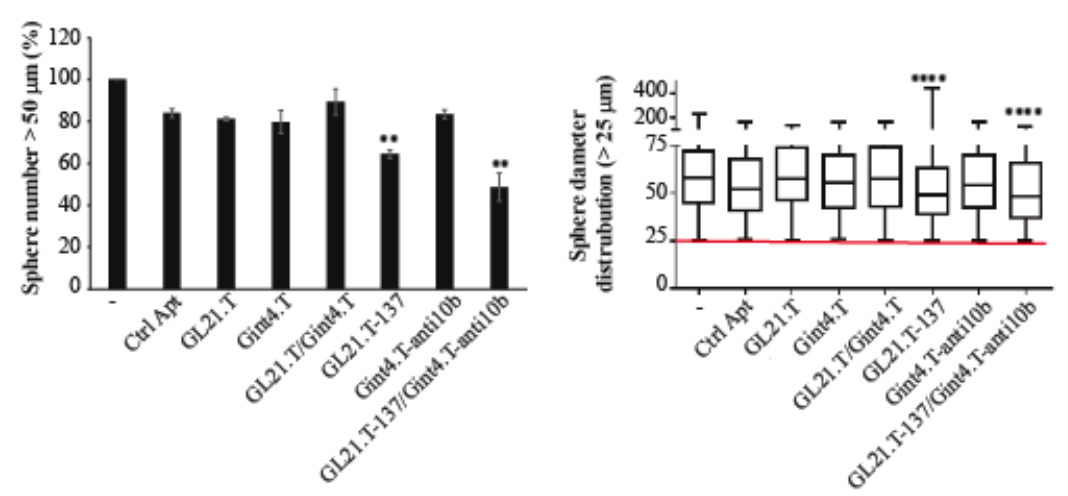

Fig. 5 
A

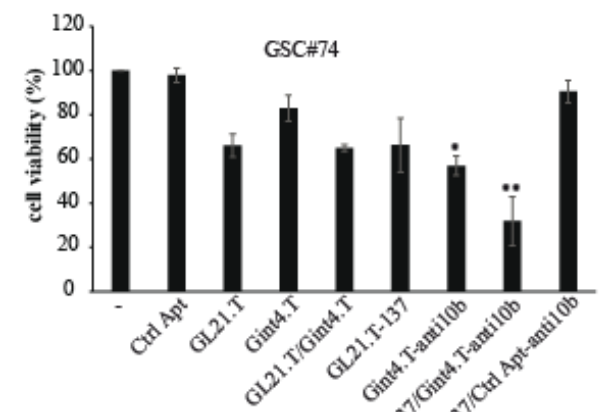

C

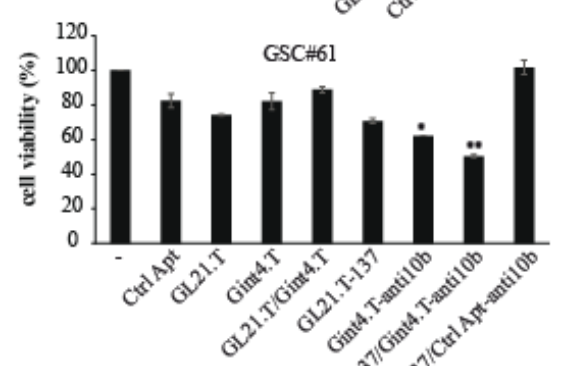

D

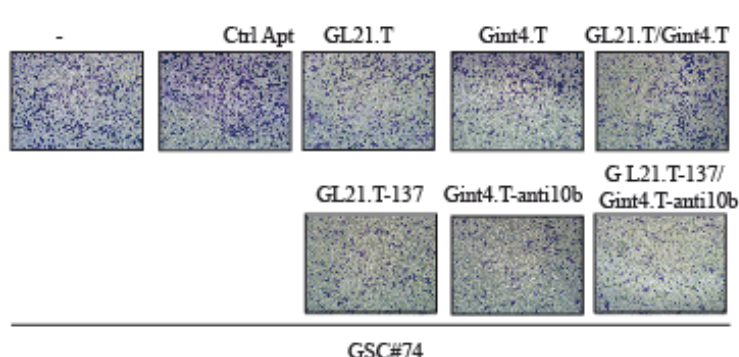

E

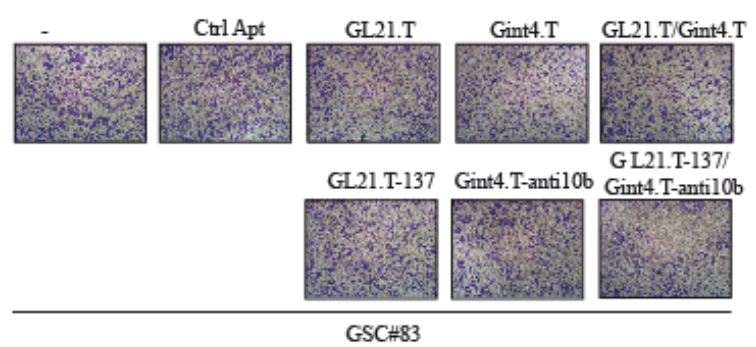

F
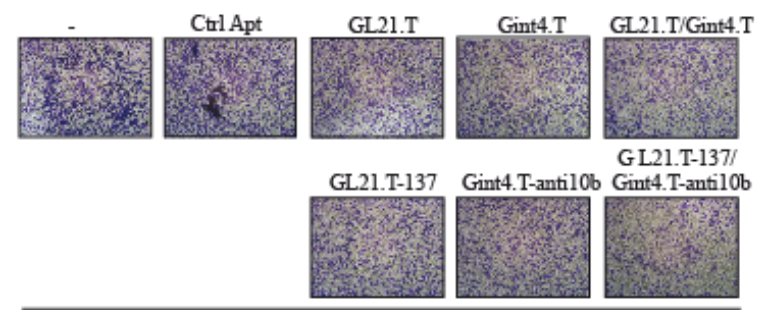

GSC\#61
B
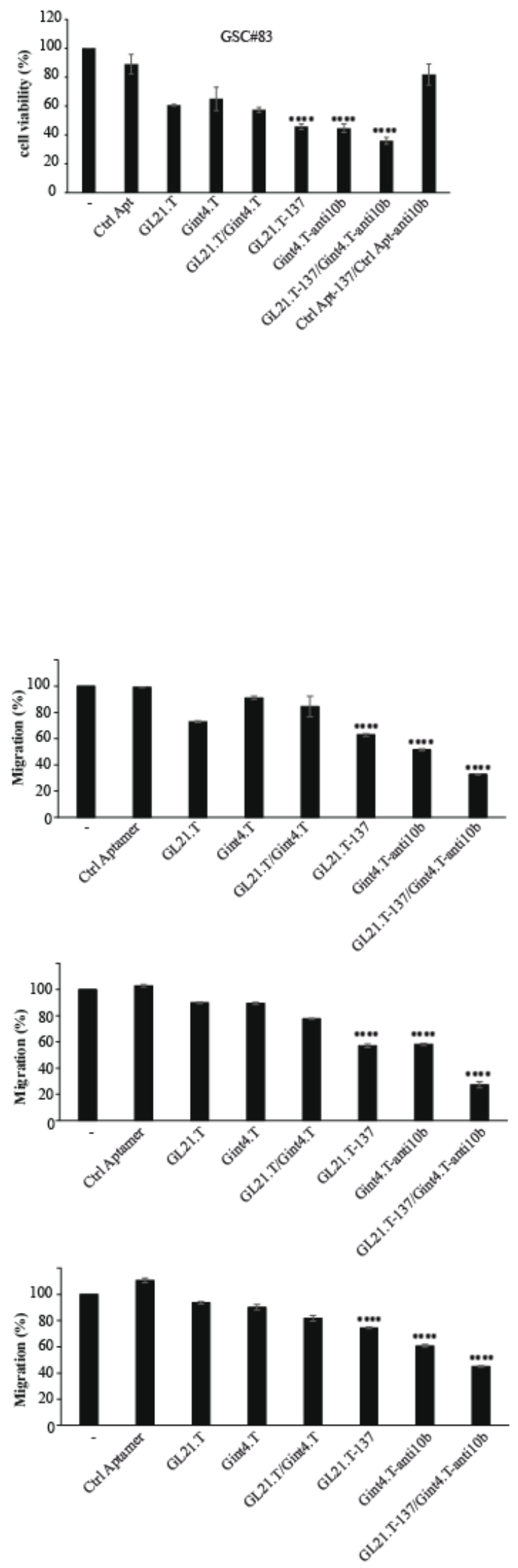

Fig. 6 


\section{BBB tri-culture model is permeable to conjugates}

Major hurdles that must be addressed in the development of RNA-based therapeutics for gliomas include the resistance of RNA molecules to enzymatic degradation in circulating fluids and penetration through the tight cerebrovascular endothelium that constitutes a physical barrier for the passage of macromolecules. We thus first evaluated the stability of each conjugate by incubation in $80 \%$ human serum for increasing times up to one week. Serum-RNA samples were recovered at the indicated time-points and analyzed by non-denaturing polyacrylamide gel electrophoresis (Fig. 7A). As shown, the conjugates were all stable up to approximately $8 \mathrm{~h}$ and then gradually degraded. The multiple RNA bands observed at longer times in antimiR-10b conjugates likely reflect the different stability of the single chain component in the conjugates. We next took advantage of an in vitro cell model to measure the ability of aptamer conjugates to cross the BBB. Short-term tri-cultures of human endothelial cells, astrocytes and pericytes grown on a three-dimensional scaffold were measured for their trans-endothelial electrical resistance (TEER) to confirm the tightness of the barrier [33]. Evans Blue was incubated in the apical chamber to check for any leaks in the model prior to and after addition of aptamer (Fig. 7B). A decrease in the TEER values typically indicated an interaction with the actin-myosin tight junction proteins that contributed to the restrictive properties of the endothelial barrier [34]. The increase in the permeability is due to the loss of adhesive properties of the tight junction proteins, which may occur during the reorganization of the actin cytoskeleton following membrane alterations during endocytosis [35, 36]. As shown in Fig. 7C, a scrambled sequence did not cause a change in the physical barrier as TEER remained relatively constant at $300 \Omega /$ $\mathrm{cm}^{2}$, while the in vitro BBB model was permeable to both GL21.T and Gint4.T aptamers, either as single molecules or conjugated (to miR-137 or anti-miR-10b respectively), as indicated by a transient drop in TEER values between $30 \mathrm{~min}$ to $1 \mathrm{~h}$ which was gradually restored by $6 \mathrm{~h}$ and was seen to be stable at $24 \mathrm{~h}$. To confirm penetration of the BBB, aptamer molecules were FAM-labelled and the fluorescence of medium was sampled at different time points from the basolateral side of the transwell. As shown in Fig. $7 \mathrm{C}$, the fluorescence measurements confirm that the BBB tri-culture model was permeable to aptamers and conjugates but not to a scrambled aptamer sequence. The Gint4.T and GL21.T aptamer appeared to show increased BBB permeability relative to the corresponding conjugates as measured by increased fluorescence, hence higher aptamer concentration in the basolateral compartment. Some transmembrane receptors, including transferrin, have been shown to promote transcytosis of bound ligands and antibodies through endothelial cells enabling them to cross the BBB [37]. We have therefore determined whether the aptamer target RTKs Axl and PDGFR $\beta$ were expressed 
in the endothelial cells, astrocytes and pericytes used for the BBB model. Indeed from the literature [38] aptamers would be typically classed as low permeability compounds (Papp $<60 \mathrm{x}$ $10^{-6} \mathrm{~cm} / \mathrm{min}$ ) based on the FDA Biopharmaceutics Classification System [39], a likely explanation for permeability would be that aptamers cross the BBB by transcytosis mediated by their target RTKs. To gain further insight on this possibility, by immunoblot we analyzed the expression of Axl and PDGFR $\beta$ in the cell types present in the tri-culture model. As shown in Fig. 7D, both PDGFR $\beta$ and Axl, receptors were expressed in endothelial cells and pericytes, but not in the astrocytes. In the tri-culture, expression of PDGFR $\beta$ and Axl receptors was higher than the endothelial and pericyte monocultures. In conclusion, the aptamer conjugates, but not a scrambled control, have the ability to cross a tri-culture cell barrier likely by a receptormediated mechanism making these aptamers promising carrier candidates for targeting brain tumors. 
A
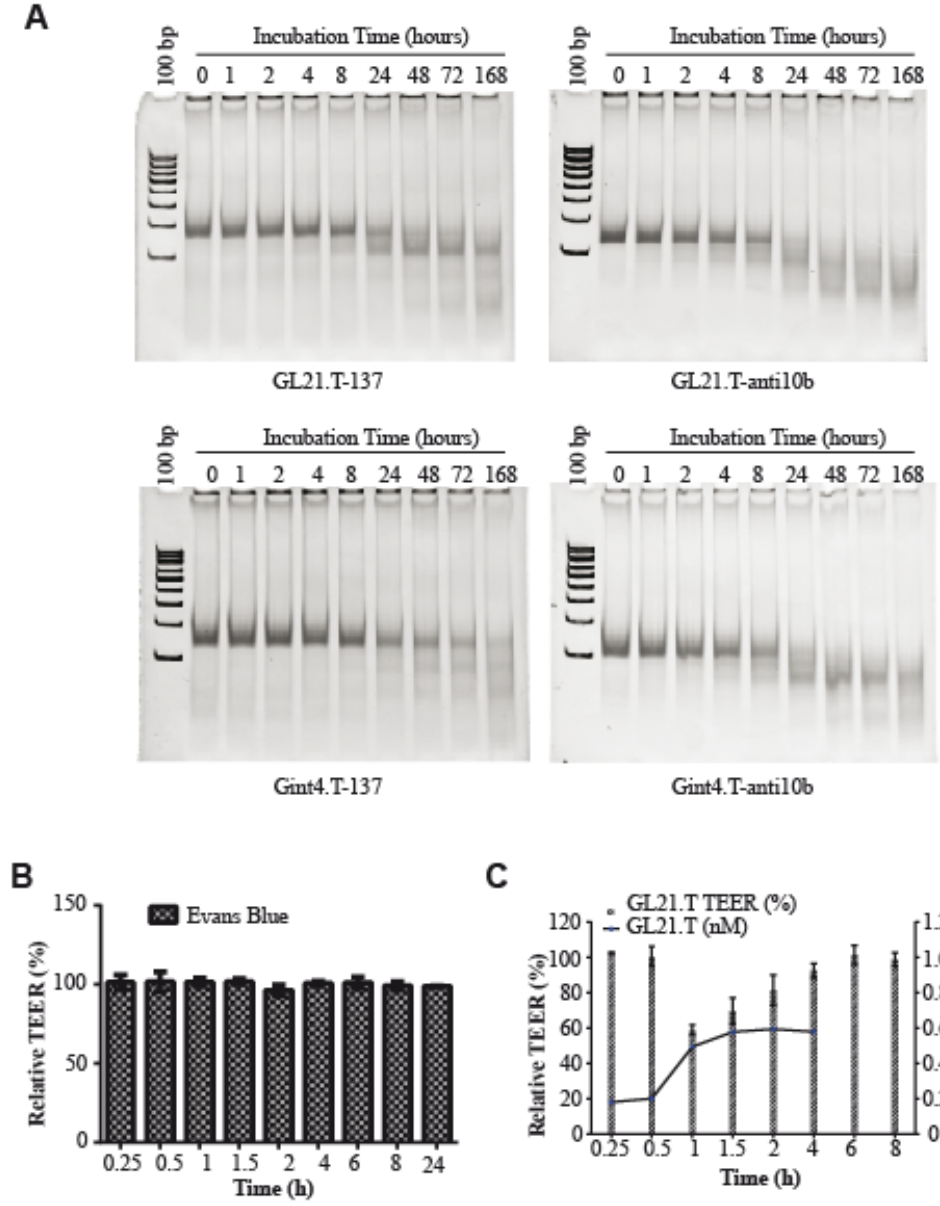

C
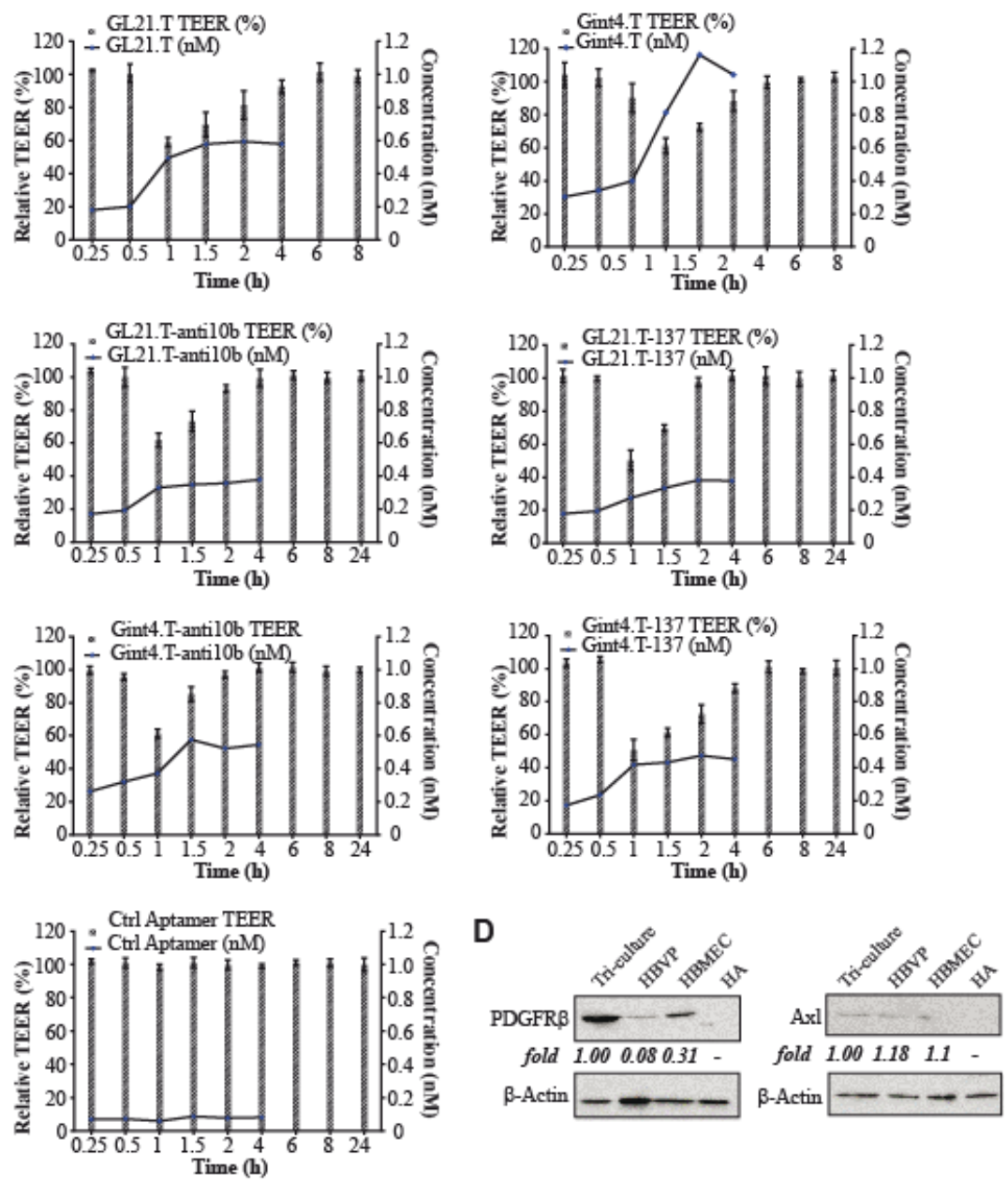

Fig. 7 


\section{Discussion}

In this study, we addressed the targeting of GBM tumor-initiating/stem-like cell population by the combined action of multiple miRNA-based therapeutics and inhibition of RTKs as a mechanismbased therapeutic strategy to combat this cancer. Several different strategies are currently being investigated to modulate (either reducing or restoring) the expression of miRNAs, including antisense, miRNA sponges or replacing therapies. Efficacy of treatments is however hampered by the lack of effective and safe delivery modalities [16].

To target the GSC population, here we used two well characterized nuclease-resistant RNA aptamers against two RTKs, AxI and PDGFRß, which are frequently highly expressed in GSCs. These aptamers have the double advantage to 1) inhibit target receptor kinase activity with the consequential downregulation of the intracellular pathways governing the survival of the target cells; and 2) be suitable carriers for the intracellular delivery of miRNA/antimiR molecules.

Indeed, it has recently been shown that the expression of multiple miRNAs is deregulated in GBM governing the main processes of this highly aggressive brain tumor, including neoangiogenesis, invasion, and resistance to apoptosis [16, 40, 41]. Furthermore, several miRNAs are differentially expressed in glioblastoma stem cells and normal neural stem cells and shown to be implicated in maintenance and propagation of the GSCs by regulating malignancy, differentiation and fate determination [42].

Here we focused on two miRNAs that have been implicated in GSC propagation: the miR-137, downregulated in GSCs, considered as a putative tumor suppressor miRNA implicated in cell cycle arrest and neuronal differentiation in primary GBM [43] and miR-10b considered an oncomiR required for GSC self-renewal and proliferation [18]. Since the aptamer conjugates are internalized through a receptor mediated manner whose effectiveness is thus limited by the amount of available receptor present on the cell surface [21], we conjugated the miR-137 and the single chain miR-10b antagonist to two different aptamers in order to deliver them separately into the target cell by targeting two distinct RTKs. This approach has the double advantage to overcome the competition of conjugates for the same target receptor and to increase the selectivity of targeting of the miRNA/antimiR combination for cells that overexpress both receptors. On the other hand, the levels of PDGFR $\beta$ are high in stem-like tumorspheres (Supplementary Fig. 1), but are significantly decreased following the combined treatments (Fig. 3 ), while the levels of $A X L$ remain high in the residual adherent-differentiated spheroids. Therefore, we conjugated the GL21.T aptamer to the miR137 that exhibited a stronger inhibitory effect on cell cycle and survival, and Gint4.T to the antimiR-10b. 
To demonstrate the effectiveness of this approach we first used tumorspheres derived from U87MG cells. When grown in 3-dimensional cultures as spheroids U87MG cells expressed the transcription factors Sox-2 and Nanog, which are involved in maintaining the pluripotency of stem-cells, and an increased expression of PDGFR $\beta$, downregulated the neuronal differentiation [44]. Further, in the spheroids the levels of miR-137 are downregulated while those of miR-10b upregulated as described for GSCs [15, 18].

Since the U87MG tumorspheres express both AxI and PDGFR $\beta$, they are a good target for the combined treatment with the GL21.T-miR137 and the Gint4.T-anti10b conjugates. As predicted from the transfection experiments, the combined treatments repress the stem-like phenotype of tumorspheres by decreasing the expression of Sox-2 and Nanog and by drastically hampering the formation of spheroids. Although each conjugate by its own is able to interfere with spheroid formation, the two conjugates strongly synergize reducing the median size of spheres. Further, the rare and small spheroids still remaining upon two weeks of combined treatment are essentially constituted by differentiated cells with poor PDGFR $\beta$ and sustained GFAP and TUBB-3 expression. The increased per cent of cells in apoptosis likely underpins the reduced number of viable cells per spheroid. As such, the combined treatment converted stem-like large undifferentiated spheroids that expressed Axl and PDGFRß, into differentiated adherent-like cells unable to grow as spheroids.

As mentioned above, when the contribution of each conjugate to the final spheroid phenotype is combined, there appears to be qualitative equivalence indicating that the biological processes engaged largely superpose. As expected, we found that the gene expression variations induced by the combined treatment result from the synergic effects of each conjugate, however, for the specific gene set analyzed, the contribution of the GL21.T-miR137 is clearly predominant over that of the Gint4.T-anti10b conjugate. Indeed, as previously reported, the GSCs share a common characteristic miRNA expression profile [42] suggesting that a reduced number of miRNAs act in concert to modulate the expression of large sets of genes that ultimately converge to propagate the stem-cell like glioblastoma cell population. Furthermore, recent reports have highlighted the involvement of miRNA deregulation in maintaining the GSC hypoxic microenvironment $[45,46]$.

Notably, the results obtained in U87MG tumorspheres were then validated with patient derived GSCs, by using three primary cell lines derived from patient tumors with GBM, of classical, and mesenchymal subtypes. Results obtained showed drastic inhibition of self-renewal and migration driven by the combined treatment that was irrespective of the tumor subtype. While cell viability and migration were comparable in the three cell lines, in the classical GSC\#61, 
inhibition of tumorsphere formation was less marked than in the GSC\#74 and in the mesenchymal GSC\#83. This is hardly imputable to the minor amount of AxI in GSC\#61, since the Gint4-anti10b, was a less efficient inhibitor of sphere formation and the amounts of cell surface receptor were similar among the three cell lines. Plausible explanations for the refractoriness of GSC\#61 to treatment may be attributable to either the other pathway that would compensate the restoration of miR-137 and miR-10b intracellular levels $[17,32]$ or because of a lower rate of recycling of the receptors.

A serious impediment to the development of macromolecular therapeutic compounds ( $>1000$ $\mathrm{Da}$ ) for brain tumors has been constituted by the presence of the BBB that hampers the transport of large peptides and nucleic acids through the cerebrovascular endothelium. To date, strategies have been developed to transport large therapeutic molecules through the BBB involving receptor-mediated mechanisms, as transferrin and insulin receptors, or loaded nanocarriers [16, 47, 48]. Most likely involving an endocytosis-based mechanism, aptamers able to cross the BBB have been recently described as targeting agents for functionalized nanoparticles [49]. Given that both AxI and PDGFR $\beta$ are expressed in vascular endothelial cells, we thus determined whether the conjugates might be able to cross an in vitro reconstituted model of BBB in a receptor-dependent manner. The cell model well reflects the poor permeability of the BBB to macromolecules and as expected also impedes the transport of a scrambled aptamer molecule. Therefore, the passage observed for the two labelled GL21.T and Gint4.T aptamers, or their conjugates, and the corresponding decrease in TEER is plausibly owed to a receptor mediated mechanism that promotes endocytosis and transcytosis. Even though in vitro evaluation of CNS targeting via BBB permeability cannot be a replacement for in vivo evaluation of CNS delivery, the in vitro BBB has enabled selection of aptamers and conjugates based on ranking those with specific transport features [34].

In conclusion, these results show the possibility of designing a mechanism-based combined cytotoxic therapy to target the elusive tumor-initiating GSC population. In this context, the GL21.T and Gint4.T aptamers play the double role of selective carriers for receptor-expressing cells and of functional inhibitors for target RTK activity (AxI and PDGFR $\beta$ respectively). Therefore, combined with the regulatory function of the conjugated miRNA-based molecules treatment results in high cell target specificity (because binding to two distinct RTKs is required) and robust therapeutic efficacy (because of their convergent action on key oncogenic pathways). Further, targeted delivery via RNA aptamers alleviates the safety of treatments also enhanced by the use of chemically modified nucleotides, which minimize nonspecific immunostimulatory effects [23]. Therefore, aptamer conjugates that are able to block 
propagation and self-renewal eluding their intrinsic refractoriness to conventional therapeutics would represent a safe and precise therapeutic option for the GSC population.

Combining RNAi to achieve a synergistic antitumor effect is an emerging therapeutic field for several cancer types, including ovarian and non-small lung cancers $[50,51]$. Nevertheless, to date the available delivery options are mostly confined to liposome-encapsulated RNAi that are selectively trapped in the liver. Therefore, the use of multiple targeting aptamers to increase the selectivity and the cytotoxicity of RNA therapeutics foresee a broad applicability to more refractory and aggressive solid cancers.

\section{Conclusions}

In conclusion, we described the development of a novel combined therapeutic strategy to target the glioma stem-like cell population through the selective delivery of multiple RNA-based conjugates. To this end, we used as molecular carriers two RNA aptamers that bind to and inhibit the receptors tyrosine kinase, AxI and PDGFRB, and, as therapeutic cargoes, the microRNA (miR-137) and micro-RNA inhibitor (antimiR-10b). We demonstrated that the effective delivery of miRNA- therapeutics to the glioma stem cell population resulted in the inhibition of GSC propagation. Importantly, we showed that the aptamer conjugates are transported by a receptor-dependent mechanism through an in vitro blood-brain barrier triculture model. By the rational design of a new mechanism-based approach, these results offer the blueprint for an effective and safe therapeutic option with great potentiality for the still unmet glioma cancer treatment.

\section{Acknowledgements}

We wish to thank L. Baraldi and F. Moscato for technical assistance and G. Condorelli for suggestions.

Funding: This work was supported by funds from: AIRC \# 13345 and 9980 (VdF), AIRC \#15584 (LRV); from the Italian Ministry of Economy and Finance to the CNR for the Project FaReBio di Qualità (VdF); Italian Ministry of Health, GR-2011-02352546 (CLE) and Brain Tumour NorthWest and In Between Ears Charity.

\section{Author contributions}


CLE designed and performed the majority of the experiments, interpreted results and assisted with manuscript preparation. $\mathrm{AR}, \mathrm{SN}$ and $\mathrm{SC}$ performed and/or assisted with several experiments. JEA, SAK, CLL and LS performed and interpreted the BBB experiments and assisted with manuscript preparation. LRV and RP provided primary GSC lines, contributed to experiments with GSCs and assisted with manuscript preparation. VdF provided intellectual input, coordinated the research, secured the funding and guided experimental studies and the preparation of the manuscript.

\section{Conflict of interest}

The authors declare no conflict of interest 


\section{References}

1. D.N. Louis, H. Ohgaki, O.D. Wiestler, W.K. Cavenee, P.C. Burger, A. Jouvet, B.W. Scheithauer, P. Kleihues, The 2007 WHO classification of tumours of the central nervous system. Acta Neuropathol. 114 (2007) 97-109.

2. R. Stupp, M.E. Hegi, W.P. Mason, M.J. van den Bent, M.J. Taphoorn, R.C. Janzer, S.K. Ludwin, A. Allgeier, B. Fisher, K. Belanger, P. Hau, A.A. Brandes, J. Gijtenbeek, C. Marosi, C.J. Vecht, K. Mokhtari, P. Wesseling, S. Villa, E. Eisenhauer, T. Gorlia, M. Weller, D. Lacombe, J.G. Cairncross, R.O. Mirimanoff; European Organisation for Research and Treatment of Cancer Brain Tumour and Radiation Oncology Groups; National Cancer Institute of Canada Clinical Trials Group, Effects of radiotherapy with concomitant and adjuvant temozolomide versus radiotherapy alone on survival in glioblastoma in a randomised phase III study: 5-year analysis of the EORTC-NCIC trial. Lancet Oncol. 10 (2009) 459-66.

3. A.J. Terzis, S.P. Niclou, U. Rajcevic, C. Danzeisen, R. Bjerkvig, Cell therapies for glioblastoma. Expert Opin. Biol. Ther. 6 (2006) 739-49.

4. H.S. Phillips, S. Kharbanda, R. Chen, W.F. Forrest, R.H. Soriano, T.D. Wu, A. Misra, J.M. Nigro, H. Colman, L. Soroceanu, P.M. Williams, Z. Modrusan, B.G. Feuerstein, Aldape K., Molecular subclasses of high-grade glioma predict prognosis, delineate a pattern of disease progression, and resemble stages in neurogenesis. Cancer Cell 9: (2006) 157-73.

5. R.G. Verhaak, K.A. Hoadley, E. Purdom, V. Wang, Y. Qi, M.D. Wilkerson, C.R. Miller, L. Ding, T. Golub, J.P. Mesirov, G. Alexe, M. Lawrence, M. O'Kelly, P. Tamayo, B.A. Weir, S. Gabriel, W. Winckler, S. Gupta, L. Jakkula, H.S. Feiler, J.G.,Hodgson C.D. James, J.N. Sarkaria, C. Brennan, A. Kahn, P.T. Spellman, R.K. Wilson, T.P. Speed, J.W. Gray, M. Meyerson, G. Getz, C.M. Perou, D.N. Hayes; Cancer Genome Atlas Research Network, Integrated genomic analysis identifies clinically relevant subtypes of glioblastoma characterized by abnormalities in PDGFRA, IDH1, EGFR, and NF1. Cancer Cell 17 (2010) 98-11.

6. S. Bozdag, A. Li, M. Baysan, H.A. Fine, Master Regulators, Regulatory Networks, and Pathways of Glioblastoma Subtypes. Cancer Inform. 13 (2014) 33-44.

7. S. Bao, Q. Wu, R.E. McLendon, Y. Hao, Q. Shi, A.B. Hjelmeland, M.W. Dewhirst, D.D. Bigner, J.N. Rich, Glioma stem cells promote radioresistance by preferential activation of the DNA damage response. Nature 444 (2006) 756-60.

8. J. Chen, Y. Li, T.S. Yu, R.M. McKay, D.K. Burns, S.G. Kernie, L.F. Parada, A restricted cell population propagates glioblastoma growth after chemotherapy. Nature. 488 (2012) 522-6. 
9. A. Eramo, L. Ricci-Vitiani, A. Zeuner, R. Pallini, F. Lotti, G. Sette, E. Pilozzi, L.M. Larocca, C. Peschle, R. De Maria, Chemotherapy resistance of glioblastoma stem cells. Cell Death Differ. 13 (2006) 1238-41.

10. J.D. Lathia, S.C. Mack, E.E. Mulkearns-Hubert, C.L. Valentim, J.N. Rich, Cancer stem cells in glioblastoma. GenesDev. 29 (2015) 1203-17.

11. F. Ohka, A. Natsume, T. Wakabayashi, Current trends in targeted therapies for glioblastoma

multiforme. Neurol Res Int. 2012 (2012) 878425.

12. D.P. Bartel, MicroRNAs: genomics, biogenesis, mechanism, and function. Cell. 116 (2004) 281-97.

13. A.L. Kasinski, F.J. Slack, MicroRNAs en route to the clinic: progress in validating and targeting microRNAs for cancer therapy. Nat. Rev. Cancer 11 (2011) 849-64.

14. M.F. Lang, S. Yang, C. Zhao, G. Sun, K. Murai, X. Wu, J. Wang, H. Gao, C.E. Brown, X. Liu, J. Zhou, L. Peng, J.J. Rossi, Y. Shi, Genome-wide profiling identified a set of miRNAs that are differentially expressed in glioblastoma stem cells and normal neural stem cells. PLoS One 7 (2012) e36248.

15. A. Bier, N. Giladi, N. Kronfeld, H.K. Lee, S. Cazacu, S. Finniss, C. Xiang, L. Poisson, A.C. deCarvalho, S. Slavin, E. Jacoby, M. Yalon, A. Toren, T. Mikkelsen, C. Brodie, MicroRNA-137 is downregulated in glioblastoma and inhibits the stemness of glioma stem cells by targeting RTVP-1. Oncotarget. 4 (2013) 665-76.

16. L. Chen, C. Kang, miRNA interventions serve as 'magic bullets' in the reversal of glioblastoma hallmarks. Oncotarget. 6 (2015) 38628-42.

17. V. Lulli, M. Buccarelli, M. Martini, M. Signore, M. Biffoni, S. Giannetti, L. Morgante, G. Marziali, R. Ilari, A. Pagliuca, L.M. Larocca, R. De Maria, R. Pallini, L. Ricci-Vitiani, miR-135b suppresses tumorigenesis in glioblastoma stem-like cells impairing proliferation, migration and self-renewal. Oncotarget. 6 (2015) 37241-56.

18. N.M. Teplyuk, E.J. Uhlmann, G. Gabriely, N. Volfovsky, Y. Wang, J. Teng, P. Karmali, E. Marcusson, M. Peter, A. Mohan, Y. Kraytsberg, R. Cialic, E.A. Chiocca, J. Godlewski, B. Tannous, A.M. Krichevsky, Therapeutic potential of targeting microRNA-10b in established intracranial glioblastoma: first steps toward the clinic. EMBO Mol Med. 8 (2016) 268-87.

19. J. Zhou, J.J. Rossi, Cell-type-specific aptamer-functionalized agents for targeted disease therapy. Mol. Ther. Nucleic Acids 3 (2014) e169.

20. J.P. Dassie, X.Y. Liu, G.S. Thomas, R.M. Whitaker, K.W. Thiel, K.R. Stockdale, D.K. Meyerholz, A.P. McCaffrey, J.O. 2nd McNamara, P.H. Giangrande, Systemic administration of 
optimized aptamer siRNA chimeras promotes regression of PSMA-expressing tumors. Nat Biotechnol 27 (2009) 839-49.

21. C.L. Esposito, L. Cerchia, S. Catuogno, G. De Vita, J.P. Dassie, G. Santamaria, P. Swiderski, G. Condorelli, P.H. Giangrande, V. de Franciscis, Multifunctional aptamer-miRNA conjugates for targeted cancer therapy. Mol. Ther. 22 (2014) 1151-63.

22. S. Catuogno, A. Rienzo, A. Di Vito, C.L. Esposito, V. de Franciscis, Selective delivery of therapeutic single strand antimiRs by aptamer-based conjugates. J Control Release 210 (2015) 147-59.

23. A.D. Keefe, S. Pai, A. Ellington, Aptamers as therapeutics. Nat Rev Drug Discov. 9 (2010) 537-50.

24. D. Xiang, C. Zheng, S.F. Zhou, S. Qiao, P.H.Tran, C. Pu, Y. Li, L. Kong, A.Z. Kouzani, J. Lin, K. Liu, L. Li, S. Shigdar, W. Duan, Superior Performance of Aptamer in Tumor Penetration over Antibody: Implication of Aptamer-Based Theranostics in Solid Tumors. Theranostics. 5 (2015) 1083-97.

25. L. Cerchia, C.L. Esposito, S. Camorani, A. Rienzo, L. Stasio, L. Insabato, A. Affuso, V. de Franciscis, Targeting AxI with an high-affinity inhibitory aptamer. Mol. Ther. 20 (2012) 2291303.

26. S. Camorani, C.L. Esposito, A. Rienzo, S. Catuogno, M. Iaboni, G. Condorelli, V. de Franciscis, L. Cerchia, Inhibition of receptor signaling and of glioblastoma-derived tumor growth by a novel PDGFRß aptamer. Mol. Ther. 22 (2014) 828-41.

27. M. Hutterer, P. Knyazev, A. Abate, M. Reschke, H. Maier, N. Stefanova, T. Knyazeva, V. Barbieri, M. Reindl, A. Muigg, H. Kostron, G. Stockhammer, A. Ullrich, Axl and growth arrestspecific gene 6 are frequently overexpressed in human gliomas and predict poor prognosis in patients with glioblastoma multiforme. Clin Cancer Res. 14 (2008) 130-8.

28. D. Akhavan, A.L. Pourzia, A.A. Nourian, K.J. Williams, D. Nathanson, I. Babic, G.R. Villa, K. Tanaka, A. Nael, H. Yang, J. Dang, H.V. Vinters, W.H. Yong, M. Flagg, F. Tamanoi, T. Sasayama, C.D. James, H.I. Kornblum, T.F. Cloughesy, W.K. Cavenee, S.J. Bensinger, P.S. Mischel, De-repression of PDGFR $\beta$ transcription promotes acquired resistance to EGFR tyrosine kinase inhibitors in glioblastoma patients. Cancer Discov. 3 (2013) 534-47.

29. K. Rubin, A. Tingström, G.K. Hansson, E. Larsson, L. Rönnstrand, L. Klareskog, L. Claesson-Welsh, C.H. Heldin, B. Fellström, L. Terracio, Induction of B-type receptors for platelet-derived growth factor in vascular inflammation: possible implications for development of vascular proliferative lesions. The Lancet 331 (1988) 1353-56. 
30. M.W. Majesky, M.A. Reidy, D.F. Bowen-Pope, C.E. Hart, J.N. Wilcox, S.M. Schwartz, PDGF ligand and receptor gene expression during repair of arterial injury. J Cell Biol 111 (1990) 2149-58.

31. R. Pallini, L. Ricci-Vitiani, G.L. Banna, M. Signore, D. Lombardi, M. Todaro, G. Stassi, M. Martini, G. Maira, L.M. Larocca, R. De Maria, Cancer stem cell analysis and clinical outcome in patients with glioblastoma multiforme. Clin Cancer Res. 14 (2008) 8205-12.

32. G. Marziali, M. Signore, M. Buccarelli, S. Grande, A. Palma, M. Biffoni, A. Rosi, Q.G. D'Alessandris, M. Martini, L.M. Larocca, R. De Maria, R. Pallini, L. Ricci-Vitiani , Metabolic/Proteomic Signature Defines Two Glioblastoma Subtypes With Different Clinical Outcome. Sci Rep. 6 (2016) 21557.

33. S. Kumar, L. Shaw, C.L. Lawrence, R.W. Lea, J.E. Alder, Developing a physiologically relevant blood brain barrier model for the study of drug disposition in glioma. Neuro-oncology 16 (2014) vi8.

34. D.B. Stanimirovic, M. Bani-Yaghoub, M. Perkins, A.S. Haqqani, Blood-brain barrier models: in vitro to in vivo translation in preclinical development of CNS-targeting biotherapeutics. Expert Opin Drug Discov. 10 (2015) 141-55.

35. G. Bazzoni, Endothelial tight junctions: permeable barriers of the vessel wall. Thromb Haemost. 95 (2006) 36-42.

36. K. Tsujita, S. Suetsugu, N. Sasaki, M. Furutani, T. Oikawa, T. Takenawa, Coordination between the actin cytoskeleton and membrane deformation by a novel membrane tubulation domain of PCH proteins is involved in endocytosis. J Cell Biol. 172 (2006) 269-79.

37. R. Gabathuler, Approaches to transport therapeutic drugs across the blood-brain barrier to treat brain diseases. Neurobiol Dis. 37 (2009) 48-57.

38. S. Aptekar, M. Arora, C.L. Lawrence, R.W. Lea, K. Ashton, T. Dawson, J.E. Alder, L. Shaw, Selective Targeting to Glioma with Nucleic Acid Aptamers. PLoS One 10 (2015) e0134957.

39. V.P. Shah, G.L. Amidon, G.L. Amidon, H. Lennernas, V.P. Shah, and J.R. Crison. A theoretical basis for a biopharmaceutic drug classification: the correlation of in vitro drug product dissolution and in vivo bioavailability Pharm Res 12, 413-420, 1995--backstory of BCS. AAPS J. 16 (2014) 894-8.

40. R.L. Skalsky, B.R. Cullen, Reduced expression of brain-enriched microRNAs in glioblastomas permits targeted regulation of a cell death gene. PLoSOne 6 (2011) e24248.

41. M. Mizoguchi, Y. Guan, K. Yoshimoto, N. Hata, T. Amano, A. Nakamizo, T. Sasaki, MicroRNAs in Human Malignant Gliomas. J Oncol. 2012 (2012) 732874. 
42. D.L. Schonberg, D. Lubelski, T.E. Miller, J.N. Rich, Brain tumor stem cells: Molecular characteristics and their impact on therapy. Mol Aspects Med. 39 (2014) 82-101.

43. J. Silber, D.A. Lim, C. Petritsch, A.I. Persson, A.K. Maunakea, M. Yu, S.R. Vandenberg, D.G. Ginzinger, C.D. James, J.F. Costello, G. Bergers, W.A. Weiss, A. Alvarez-Buylla, J.G. Hodgson, MiR-124 and miR-137 inhibit proliferation of glioblastoma multiforme cells and induce differentiation of brain tumor stem cells. BMC Med. 6 (2008) 14.

44. Y. Kim, E. Kim, Q. Wu, O. Guryanova, M. Hitomi, J.D. Lathia, D. Serwanski, A.E. Sloan, R.J. Weil, J. Lee, A. Nishiyama, S. Bao, A.B. Hjelmeland, J.N. Rich, Platelet-derived growth factor receptors differentially inform intertumoral and intratumoral heterogeneity. Genes Dev. 26 (2012) 1247-62.

45. J. Hu, T. Sun, H. Wang, Z. Chen, S. Wang, L. Yuan, T. Liu, H.R. Li, P. Wang, Y. Feng, Q. Wang, R.E. McLendon, A.H. Friedman, S.T. Keir, D.D. Bigner, J. Rathmell, X.D. Fu, Q.J. Li, H. Wang, X.F. Wang, MiR-215 is Induced Post-transcriptionally via HIF-Drosha Complex and Mediates Glioma-Initiating Cell Adaptation to Hypoxia by Targeting KDM1B. Cancer Cell 29 (2016) 49-60.

46. J. Wei, E.K. Nduom, L.Y. Kong, Y. Hashimoto, S. Xu, K. Gabrusiewicz, X. Ling, N. Huang, W. Qiao, S. Zhou, .C. Ivan, G.N. Fuller, M.R. Gilbert, W. Overwijk, G.A. Calin, A.B. Heimberger, MiR-138 exerts anti-glioma efficacy by targeting immune checkpoints. Neuro Oncol. (2015) pii: nov292.

47. M.J. Gomes, S. Martins, B. Sarmento, siRNA as a tool to improve the treatment of brain diseases: Mechanism, targets and delivery. Ageing Res Rev. 21 (2015) 43-54.

48. B. Milojkovic Kerklaan, O. van Tellingen, A.D. Huitema, J.H. Beijnen, W. Boogerd, J.H. Schellens, D. Brandsma, Strategies to target drugs to gliomas and CNS metastases of solid tumors. J Neurol. 263 (2015) 428-40.

49. H. Gao, J. Qian, Z. Yang, Z. Pang, Z. Xi, S. Cao, Y. Wang, S. Pan, S. Zhang, W. Wang, X. Jiang, Q. Zhang, Whole-cell SELEX aptamer-functionalized poly(ethylenglycol)-poly(ecaprolactone) nanoparticles for enhanced targeted glioblastoma therapy. Biomaterials 33 (2012) 6264-72.

50. A.L. Kasinski, K. Kelnar, C. Stahlhut, E. Orellana , J. Zhao, E. Shimer, S. Dysart, X. Chen, A.G. Bader, F.J. Slack, A combinatorial microRNA therapeutics approach to suppressing non-small cell lung cancer. Oncogene 34 (2014) 3547-55.

51. M. Nishimura, E.J. Jung, M.Y. Shah, C. Lu, R. Spizzo, M. Shimizu, H.D. Han, C. Ivan, S. Rossi, X. Zhang, M.S. Nicoloso, S.Y. Wu, M.I. Almeida, J. Bottsford-Miller, C.V. Pecot, B. Zand, K. Matsuo, M.M. Shahzad, N.B. Jennings, C. Rodriguez-Aguayo, G. Lopez-Berestein, A.K. 
Sood, G.A.Calin, Therapeutic synergy between microRNA and siRNA in ovarian cancer treatment. Cancer Discov. 3 (2013) 1302-15.

\section{Figure legends}

Fig. 1. MiR-137 and antimiR-10b combined transfection. (A-D) U87MG-derived tumorspheres were left untransfected (-) or transfected with control miRNA (Ctrl miR), control anti-miRNA (Ctrl antimiR), miR.137 or antimiR-10b alone or in combination, as indicated. (A) Following $72 \mathrm{~h}$ cell lysates were immunoblotted with antibodies against either GLIPR-1 or Bim. Anti-BActin was used to confirm equal loading. Values below the blots indicate normalized quantitation relative to untransfected. (B) After $24 \mathrm{~h}$ cell motility was analyzed and microphotographed (left panel). Quantization is expressed as per cent of migrated cells with respect to untransfected cells (right pane). (C) Following $72 \mathrm{~h}$, cell viability was measured and expressed as per cent of viable cells with respect to untransfected cells. Ctrl miR, 200 nmol/l; Ctrl antimiR, $200 \mathrm{nmol} / \mathrm{l}$; Ctrl miR/Ctrl antimiR, $100 \mathrm{nmol} / \mathrm{l}$ each; miR-137/antimiR-10b, $100 \mathrm{nmol} / \mathrm{l}$ each. (D) Tumorsphere formation were analyzed following 10 days from transfection of synthetic controls (Ctrl), miR-137, antimiR$10 \mathrm{~b}$ or combined miR-137 and antimiR-10b as indicated. Left panel, spheres with a diameter greater than $50 \mu \mathrm{m}$ were counted; right panel, representative microphotographs are shown (scale bar: $100 \mu \mathrm{m}$ ). In (B-D) Vertical bars indicate the standard deviation values. Statistics for conjugate treatments versus untrasfected sample: ${ }^{*}, p<0.05 ;{ }^{* *}, p<0.01 ;{ }^{* * *}, p<0.0001$.

Fig. 2. GL21.T and Gint4.T conjugates activity in U87MG derived tumorspheres. (A) Upper panel, scheme of miR-137 and antimiR-10b aptamer conjugates. Lower panel, scheme of the general protocol used for 10 day-treatment of tumorspheres. (B, C) Levels of Nanog, Sox-2 and PDGFR $\beta$ were analyzed by immunoblotting. Equal loading was confirmed by immunoblot with anti-Tubulin ( $(\mathrm{T} T \mathrm{ub})$ antibodies. Values below the blots: normalized quantitation relative to untreated. Ctrl aptamer: unrelated aptamer used as control; GL21.T-137: GL21.T conjugated to miR-137; GL21.T-anti10b: GL21.T conjugated to antimiR-10b; Gint4.T-137: Gint4.T conjugated to miR-137; Gint4.T-anti10b: Gint4.T conjugated to antimiR-10b. (D- F) Analyses of tumorsphere formation. (D) Spheres with a diameter greater than $50 \mu \mathrm{m}$ were counted and expressed as percentage relative to untreated sample (-) that was set to $100 \%$. (E) Representative microphotographs are shown (scale bar: $100 \mu \mathrm{m}$ ). (F) Box plot representation of diameter measures of spheres with a diameter greater than $25 \mu \mathrm{m}$ (indicated by the horizontal 
red line). In (D) Vertical bars indicate the standard deviation values. In (D, F) Statistics: * $p<$ $0.05 ;{ }^{* *}, \mathrm{p}<0.01 ;{ }^{* \star * *}, \mathrm{p}<0.0001$ (versus untreated sample).

Fig. 3. GL21.T-miR137 and Gint4.T-anti10b conjugates combined effect on U87MG stemness. (A-D) U87MG derived tumorspheres were left untreated (-) or treated as indicated in Fig. 2A (lower panel). (A) Levels of PDGFRß, Nanog, Sox-2 and Slug were analyzed by immunoblotting. Anti- $\beta$ Actin antibodies were used to confirm equal loading. Values below the blots: normalized quantitation relative to untreated. (B-D) Analyses of tumorsphere formation. (B) Spheres with a diameter greater than $50 \mu \mathrm{m}$ were counted and expressed as percentage relative to untreated sample (-). (C) Representative microphotographs are shown (scale bar: $100 \mu \mathrm{m}$ ). (D) Box plot representation of diameter measures of spheres with a diameter greater than $25 \mu \mathrm{m}$ (indicated by the horizontal red line). (E) Tumorspheres were treated as schematized and cell viability was measured and eexpressed as percentage relative to untreated sample (-, set to 100\%). (F) Levels of GFAP and TUBB3 were measured by RT-qPCR in U87MG derived tumorspheres left untreated (-) or treated for 10 days with indicated aptamers or conjugates as in Fig. 2A. In (B, D and E) Statistics for conjugate treatments versus untreated sample: ${ }^{*}, \mathrm{p}<0.05 ;{ }^{* *}, \mathrm{p}<0.01{ }^{* * *}, \mathrm{p}<0.0001$. In $(\mathrm{B}, \mathrm{E}$ and $\mathrm{F})$ Vertical bars indicate the standard deviation values.

Fig. 4. GL21.T-miR137 and Gint4.T-anti10b conjugates combined effect on cell proliferation and migration. (A, B) U87MG derived tumorspheres were left untreated (-) or treated as in Fig. 2A. (A) Cell cycle were analyzed following PI incorporation. Differentiated 2-D growing U87MG cells were included as control. (B) Cell viability was measured and expressed as per cent of viable cells with respect untreated cells (-). (C) Annexin V/PI staining was analyzed by flow cytometry of U87MG derived tumorspheres following $72 \mathrm{~h}$ of treatment. (D, E) RT-qPCR array analysis of U87MG-derived tumorspheres treated with indicated conjugates for 7 days, renewing the treatments at day 3 and 5. A combination of control aptamer conjugated either to miR-137 or antimiR-10b (Ctrls) was used as control. (D) Genes whose levels significantly changed ( $\geq \pm 1.5$ ) are shown as heat map. (E) Gene ontology (by GOrilla) analyses of significantly changed genes. (F) Cell motility was analyzed following $24 \mathrm{~h}$ treatment. Left panel, representative microphotographs are shown. Right panel, the results were quantified and expressed as per cent of migrated with respect to untreated cells. Statistics versus untreated sample: ${ }^{\star \star \star *}, \mathrm{p}<$ 0.0001 . In (B, F) Vertical bars indicate the standard deviation values. 
Fig. 5. Combined effect of conjugates on primary GSC tumorsphere formation. (A) Absolute quantization of miR-137 and miR-10b in GSC\#74, GSC\#83 and GSC\#61. (B-D) Sphere formation of indicated primary GSC-derived tumorspheres left untreated (-) or treated as in Fig. 2A. Left panels, spheres with a diameter greater than $50 \mu \mathrm{m}$ were counted and expressed as percentage relative to untreated samples (-) set to $100 \%$. Right panels, Box plot representation of diameter measures (spheres with a diameter greater than $25 \mu \mathrm{m}$, indicated by the horizontal red line). In (A-D) Vertical bars indicate the standard deviation values. In (B-D) Statistics of conjugate treatments versus untreated samples: ${ }^{*}, p<0.05 ;{ }^{* *}, p<0.01 ;{ }^{* \star *}, p<0.001 ;{ }^{* \star * *}, p<$ 0.0001 .

Fig. 6. Combined effect of conjugates on primary GSC cell viability and migration. (A-C) Primary GSC cell viability was measured following 10 days of aptamer or conjugate treatments as in Fig. 2A. Results are expressed as per cent of viable cells with respect to untreated cells (-) set to $100 \%$. (D-F) Cell motility of indicated GSC left untreated or treated with indicated aptamers or conjugates $(400 \mathrm{~mol} / \mathrm{l})$ for $24 \mathrm{~h}$ was analyzed. Left panels, representative microphotographs are shown. Right panels, the results are expressed as per cent of migrated with respect to untreated cells. In (A-F) Vertical bars indicate the standard deviation values. Statistics of conjugates versus untreated samples: ${ }^{*}, p<0.05 ;{ }^{* *}, p<0.01 ;{ }^{* * *}, p<0.001 ;{ }^{* * *}, p<0.0001$.

Fig. 7. Conjugate serum stability and BBB permeability. (A) Analyses of serum stability of indicated conjugates. (B) Confirmation of the integrity of the BBB model prior to addition of aptamers or conjugates. Change in trans-endothelial electrical resistance measured in the presence of Evans Blue incubated in the apical chamber, relative to trans-endothelial electrical resistance in presence of media alone. (C) FAM-labelled aptamer or conjugates ability to cross the in vitro BBB model. Change in trans-endothelial electrical resistance in presence of aptamer or conjugates relative to trans-endothelial electrical resistance in presence of media alone and concentration of aptamer or conjugates measured by fluorescence from the basolateral side. (D) Axl and PDGR $\beta$ expression were analyzed BBB model tri-culture. Equal loading was confirmed by immunoblotting with anti- $\beta$ Actin antibodies. Values below the blots: normalized quantitation relative to tri-culture. 


\section{Graphical abstract}
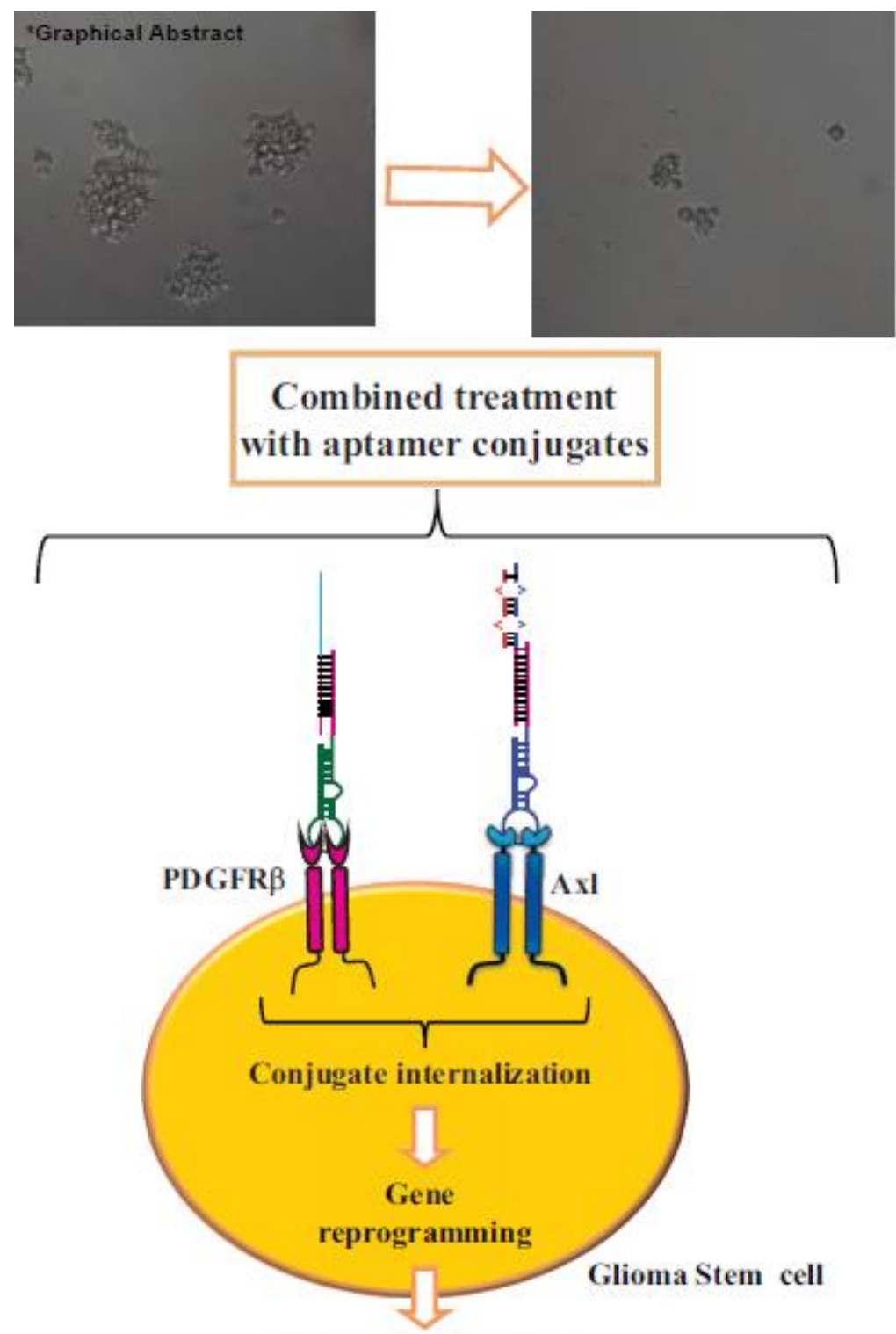

Stem cell eradication

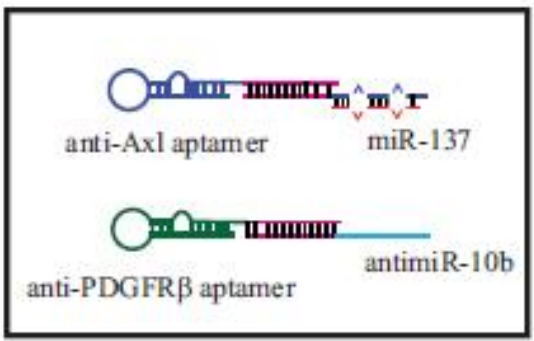

Aptamers as Trojan-Horse for microRNAs to eradicate the glioblastoma stem-like population 